\title{
Pueraria Flos Alleviates Alcoholic Liver Injury Via Regulation of PPARa and MAOA: Deciphering the Effective Forms and Potential Mechanism Based on a Bioinformatics-Integrated Metabolic Profile Strategy and Experimental Validation
}

Jialin Qu ( $\nabla$ jialin_qu@126.com )

First Affiliated Hospital of Dalian Medical University https://orcid.org/0000-0003-2020-4783

Qiuyue Chen

Shenyang Pharmaceutical University

\section{Tianfu Wei}

Dalian Medical University

Ning Dou

Shenyang Pharmaceutical University

\section{Dong Shang}

Dalian Medical University

\section{Dan Yuan}

Shenyang Pharmaceutical University

\section{Research}

Keywords: Pueraria Flos, alcoholic liver disease, effective forms, bioinformatics-integrated metabolic profile strategy, experimental validation

Posted Date: November 23rd, 2021

DOI: https://doi.org/10.21203/rs.3.rs-1055239/v1

License: (c) (i) This work is licensed under a Creative Commons Attribution 4.0 International License. Read Full License 
1 Pueraria Flos alleviates alcoholic liver injury via regulation of PPARa and

2 MAOA: deciphering the effective forms and potential mechanism based on

3 a bioinformatics-integrated metabolic profile strategy and experimental 4 validation

5

6 Jialin $\mathrm{Qu}^{\mathrm{a}}{ }^{\mathrm{a}, \mathrm{b}}$, Qiuyue Chen ${ }^{\mathrm{a}}$, TianfuWei ${ }^{\mathrm{b}}$, Ning Dou ${ }^{\mathrm{a}}$, Dong Shang ${ }^{\mathrm{b}, * *}$ and Dan Yuan ${ }^{\mathrm{a}, *}$

7

$8{ }^{a}$ Department of Traditional Chinese Medicine, Shenyang Pharmaceutical University, 103

$9 \quad$ Wenhua Road, Shenyang, 110016, China

$10{ }^{\mathrm{b}}$ Clinical Laboratory of Integrative Medicine, The First Affiliated Hospital of Dalian Medical

University, No. 222, Zhongshan Road, Dalian 116011, China

*Correspondence should be addressed to Dan Yuan; yuandan_kampo@163.com

Co-correspondence should be addressed to Dong Shang; shangdong@dmu.edu.cn 


\section{Abstract}

Background: Pueraria Flos, a representative medicinal and edible antidote for alcoholism, has rich clinical experience and remarkable curative effect in the treatment of alcoholic liver disease (ALD). However, its effective forms and hepatoprotective mechanism are remained unknown. Methods: A strategy based on UPLC-QTOF-MS combined with mass defect filtering technique was established for comprehensively identifying prototypes and metabolites absorbed and excreted into rat plasma, urine, bile and feces after oral administration. Then, the absorbed constituents with a relative high level were subjected to the network pharmacology, functional enrichment analysis and molecular docking to clarify the potential mechanism in the treatment of ALD. Furthermore, the therapeutic effect of PF on ALD and predicted mechanisms was further evaluated using a rat model of alcohol-induced liver injury and Western blot analysis. Results: 25 absorbed prototype constituents and 82 metabolites were identified or tentatively characterized with glucuronidation, sulfation, methylation, hydroxylation and reduction as their major metabolic pathways. The constructed absorbed constituent-target-pathway-disease network and docking analysis revealed that 4 metabolic components Te-7XG, genistein-7G-4'S, tectoridin-4'S and Te-7XG-4'S, 2 targets MAOA and PPARA, and 6 pathways related to lipid regulation and amino acid metabolism may play crucial roles in the PF mediated protection against ALD. An in vivo validation in rat further demonstrated that PF alleviated liver injury via activating and suppressing the PPARA and MAOA expression, respectively.

Conclusions: The present results not only increase the understanding on the effective form and molecular mechanism of PF mediated protection against ALD, but also promote better application of PF as supplement food and herbal medicine for the treatment of ALD.

\section{Keywords}

Pueraria Flos, alcoholic liver disease, effective forms, bioinformatics-integrated metabolic profile strategy, experimental validation 


\section{Introduction}

With the development of social economy, the alcoholic drinkers have been increasing in recent years. Alcohol abuse and alcoholism have emerged as a common public health problem all round the world. As an important organ responsible for alcohol metabolism, the liver is the main target organ for alcohol toxicity. Accordingly, the incidence rate of alcoholic liver disease (ALD) caused by long-term heavy drinking is increasing year by year. Therefore, exploring the effective treatment of ALD has become a particularly important issue which captures the attention of academic research. At present, the treatment of ALD has largely relied on three medications or healthproducts, namely, synthetic alcohol dehydrogenase inhibitor fomepizole, plant extracts Camette and Hydrodol tablets import from Denmark and Australia, and an extract of oyster powder known as Haiwang Jinzun tablet. However, their application is restricted due to some side effects, such as hypotension, slow heartbeat and coma, and the high price that depends on importing and safety problems incurred by the influence of fresh storage and marine pollution on raw materials. On the whole, the drugs for treatment of ALD enjoys huge market capacity and potential in two respects. First, it has a stable and huge beneficiary population. Second, it is in a situation where imported drugs are dominant and domestic new drugs are obviously scarce.

There have been some records of 'alcohol injury', 'alcohol jaundice' and 'alcoholism' in ancient Chinese medicine books for a long time. As an important part of traditional Chinese medicine, some ethnic and edible medicines have rich clinical experience and remarkable curative effect in the treatment of ALD, and they are valuable and prospective for drug research and development. Pueraria Flos (PF), known as 'Ge-hua' in Chinese, is botanically from the dried flowers of Pueraria thomsonii Benth. or Pueraria lobate (Willd.) Ohwi. As a medicinal and edible cognate, it has been traditionally used to relieve toxic symptoms caused by excessive alcohol consumption, such as hangover, nausea, headache and red face in China, Japan and Korea for over 1500 years. Consequently, PF has been considered as the most representative antidote in traditional medicine. Recent pharmacological studies showed that extracts and compounds from PF possessed various bioactivities, such as hepatoprotective [1], hypoglycemic [2], hypolipidemic [2], estrogenic [3] and anti-inflammatory effects [4]. With 
respect to the chemical constituents of $\mathrm{PF}$, more than 80 compounds have been isolated and identified to date, among which, isoflavanoids and triterpenoid saponins are two major types of constituents and play important roles in its pharmacological effects. Five isoflavanoids kakkalide, irisolidone, tectorigenin-7-O-xylosylglucoside, tectoridin and tectorigenin have been proved for potential medicinal values and regarded as important phytochemical markers for the quality evaluation and differentiation between species under PF [5].

According to the concept of 'effective forms' and 'additive effect' of pharmacodynamics substances of TCMs, only the constituents and/or metabolites that are successfully assimilated into the body circulation system and maintain a considerable concentration level by additive effect in target organs may exert curative effects. In the past decade, the metabolism of PF isoflavanoids in vitro has been reported by some scholars [6]. Our research group have also been devoting our efforts towards their ADME (absorption, distribution, metabolism, elimination) characteristic by column-separation, ultra-performance liquid chromatography/quadrupole time-of-flight mass spectrometry (UPLC-QTOF-MS) and NMR spectroscopy [7-13]. Ten urinary and biliary metabolites have been isolated and structural identified, the plasma pharmacokinetics as well as urinary and biliary excretion of the conjugated metabolites were also determined in rats after oral administration. The results indicated that glucuronidation and/or sulfation after deglycosylation at C-7 position was the major metabolic pathway of isoflavanoids from PF in vivo. Additionally, kaikasaponin III, soyasaponin I and kakkasaponin I that were the most abundant saponins in PF showed powerful protective effects against liver damage in previous study, which are also responsible for the overall curative effects of PF [14]. However, to the best of our knowledge, no reports have described the global metabolic profile of triterpenoid saponins or whole plant extract of PF in vivo. Moreover, pharmacological mechanisms and bioactive components of PF for the treatment of ALF are still not clear.

In this study, the absorbed and excreted prototypes and metabolites of the extract of Pueraria thomsonii Benth. in rat plasma, urine, bile and feces were firstly characterized by UPLC-QTOF-MS. Furthermore, with subsequent visualization of "ingredient-target-pathwaydisease" association network constructed by network pharmacology and binding interactions between key ingredients with targets performed by molecular docking simulation, the potential 
active components and underlying pharmacological mechanisms for the effect of PF on AFL were explored. Furthermore, the predicted key targets of PF against ALD were validated in an alcohol-induced liver injury rat model, which would promote better application of PF that is a medical resource for developing a supplement food or an herbal medicine for the treatment of ALD (Figure 1).

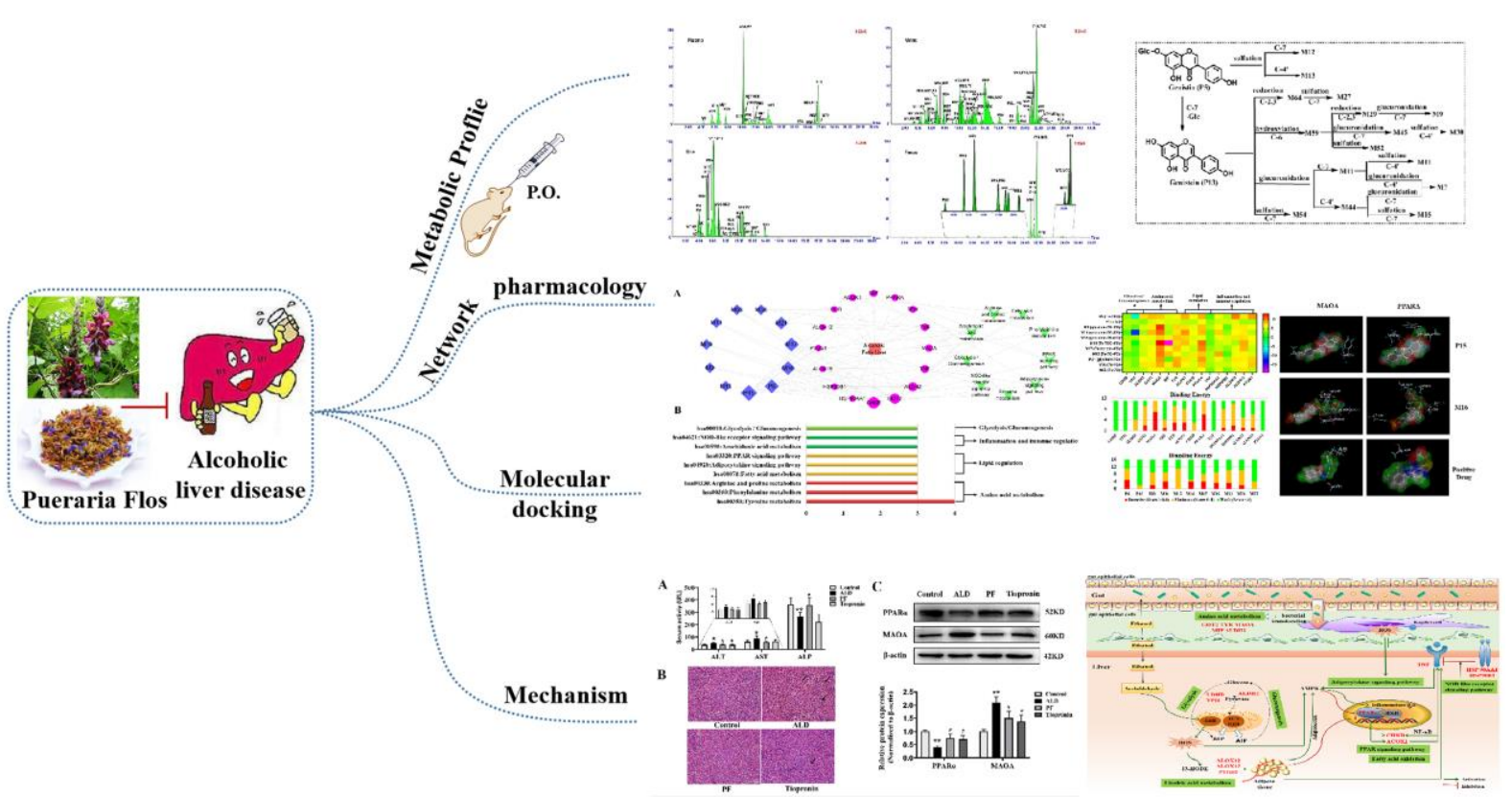

Figure 1 The schematic diagram of the present study

\section{Materials and Methods}

\subsection{Chemicals, reagents, and materials}

Pueraria Flos (Batch No.161001) collected from Anhui Province was purchased from Tongrentang TCM store (Shenyang, Liaoning Province, China) in October 2017, and was authenticated as the flower of Pueraria thomsonii Benth. by Prof. Dan Yuan (Department of Traditional Chinese Medicine, Shenyang Pharmaceutical University). A voucher specimen was deposited at the authors' laboratory. The reference compounds daidzein (16) and luteolin (20) were purchased from Funakoshi Co., Ltd. (Tokyo, Japan). Tectorigenin-7-O- $\beta$-Dxylosylglucopyranoside (10), tectoridin (12), genistein (23), tectorigenin (25) and irisolidone (32) were isolated from the extracts of the flowers of Pueraria thomsonii Benth. or Pueraria lobate (Willd.) Ohwi. in our previous studies [4]. The purities of these compounds evaluated with HPLC-photodiode array detector (PDA) were more than $95 \%$. 
HPLC-grade acetonitrile, methanol and formic acid were supplied by Fisher Scientific

Company Inc. (Fairlawn, NJ). Ultra-pure water (18.2 M $\Omega$ ) was prepared with a Milli-Q water purification system (Millipore, Milford, MA, USA). All other reagents were of analytical grade and purchased from Shandong Yuwang Pharmaceutical Co., Ltd. (Yucheng, Shandong Province, China).

Rabbit monoclonal antibodies against peroxisome proliferator-activated receptor $\alpha$ (PPARA), monoamine oxidase type A (MAOA) and $\beta$-actin were obtained from Abcam Company (Cambridge, MA, USA) and ABclonal Biotechnology (Wuhan, Hubei Province, China), respectively. Protein extraction kit were purchased from KeyGen BioTech Co., Ltd (Nanjing, Jiangsu Province, China)

\subsection{Preparation of Pueraria Flos extracts}

Dried flower of Pueraria thomsonii Benth. (1 kg) were weighed accurately and refluxextracted twice with $80 \% \mathrm{EtOH}(1: 12$ and then 1:10, w/v) for $1 \mathrm{~h}$ each time. After filtered with six-layer absorbent gauze, the two filtered extracts were combined, concentrated under vacuum to $1 \mathrm{~L}$ (equal to $1 \mathrm{~g}$ crude herb/mL) and finally transformed into the freeze-dried powder.

$50 \mathrm{mg}$ of prepared powder was dissolved again with $10 \mathrm{~mL}$ of methanol/water $(8: 2, \mathrm{v} / \mathrm{v})$ and extracted for $30 \mathrm{~min}$ under ultrasound. After centrifuged at $13000 \mathrm{rpm}$ for $10 \mathrm{~min}$ at $4{ }^{\circ} \mathrm{C}$ and filtered through a $0.22 \mu \mathrm{m}$ filter. $1.0 \mu \mathrm{L}$ of filtrate was injected to UHPLC-QTOF-MS for qualitative analysis.

To calculate the administered dose, the contents of three major ingredients were quantitatively determined by reported HPLC-UV with external standard method [15]. Results indicated that the contents of tectorigenin-7-O- $\beta$-D-xylosylglucopyranoside (10), tectoridin (12) and tectorigenin $(\mathbf{2 5})$ in the extract were $112.5,96.64$ and $19.81 \mathrm{mg} / \mathrm{g}$, respectively.

\subsection{Sample collection and pretreatment in vivo}

Nine male Sprague-Dawley rats (200 \pm 20 g body weight about 6-8-week-old) purchased from the animal center of Shenyang Pharmaceutical University were maintained in a in ambient houses $\left(22 \pm 2^{\circ} \mathrm{C}\right)$ with a 12 -h light/12-h dark cycle. To be acclimatized, rats were allowed soyfree food and water ad libitum in metabolic cages for one week before the experiments. The animals were divided into three groups at random: a dosed plasma collection group $(n=3)$; a dosed urine and feces collection group $(n=3)$ and a dosed bile collection group $(n=3)$. All 
animals were fasted for $12 \mathrm{~h}$ before the experiments and provided with free access to water and sugar over the period of sample collection. PF extracts was suspended in a $0.5 \%$ carboxy-methyl cellulose sodium salt aqueous solution with a concentration of $0.11 \mathrm{~g} / \mathrm{mL}$ and administered by oral gavage at a dose of $1.1 \mathrm{~g} / \mathrm{kg}$ body weight (equivalent to $200 \mathrm{mg}$ tectoridin per $\mathrm{kg}$ ) to rats. All experimental protocol was approved by the Ethics Review Committee for Animal Experimentation of Shenyang Pharmaceutical University (License number: SCXK (Liao) 20150001).

Serial blood samples (approximately $0.5 \mathrm{~mL}$ ) were collected from the suborbital vein and placed in heparinized polythene tubes at $0,0.5,1,1.5,2,3,4$ and $8 \mathrm{~h}$ after oral administration, then immediately centrifuged at 3,500 rpm for $10 \mathrm{~min}$ at $4{ }^{\circ} \mathrm{C}$ to obtain plasma. Urine and bile samples were respectively collected at $0-2,2-4,4-8,8-12$ and $12-24 \mathrm{~h}$ after the dosing. Feces samples were collected at $0-12$ and $12-24 \mathrm{~h}$, then left in a cool and dry place to dryness. The collected plasma, urine and bile samples were mixed and pretreated using solid phase extraction, while feces sample was extracted with ultrasonic in methanol/water (75:25, v/v) according to our previous method [13].

\subsection{UPLC-QTOF-MS analysis}

The condition of chromatographic separation and mass detection was almost the same as those reported in literature [11]. The only difference is the change of elution gradient, which was listed as follows: 0-1 min, 5\%-8\% B; 1-8 min, 8\%-13\% B; 8-9.5 min, 13\%-15\% B; 9.5$11.5 \min , 15 \%$ B; $11.5-14 \min , 15 \%-16 \%$ B; 14-18 min, $16 \%-17 \%$ B; $18-30 \mathrm{~min}, 17 \%-65 \%$ B; 30-30.5 min, 65\%-99\% B; 30.5-30.6 min, 99\%-5\% B.

\subsection{Network pharmacology analysis}

\subsubsection{Identification of candidate targets of absorbed constituents and ALD}

After identifying the absorbed and excreted ingredients of PF in vivo by UPLC-QTOFMS/MS. The chemical structure of absorbed constituents in plasma and bile with relative content higher than 3\% was obtained as SDF format by ChemBioDraw Ultra 12.0 software and then submitted to Swiss Target Prediction platform (http://www.swisstargetprediction.ch/) to predict the most probable protein targets. The official gene names of top 100 targets with high matching degrees were selected for subsequent analysis. The targets associated with "alcoholic liver disease" were acquired from OMIM, TTD, CTD, GAD, DisGeNET and GeneCards 
Database.

\subsubsection{The protein-protein interactions (PPIs) network analysis}

The Search Tool for the Retrieval of Interacting Genes (STRING) database (https://stringdb.org/) provides each predicted PPI information as well as the data which have been experimentally confirmed. The version 11.0 of STRING was applied to acquire the PPI information, with the species limited to "Homo sapiens" and a confidence score $>0.9$. These PPI targets were defined as core targets for further analysis.

\subsubsection{Enrichment analysis}

The KEGG pathway analysis was performed by Database for Annotation, Visualization and Integrated Discovery (DAVID, https://david.ncifcrf.gov/home.jsp, ver. 6.8) to find the signaling pathways related to candidate targets and then the AFL-related pathways were selected.

\subsubsection{Network construction and analysis}

The absorbed constituent-target-pathway-disease network was constructed by utilizing the network visualization software Cytoscape 3.2.1, which supplies a method for data integration, analysis and visualization for complicated network analysis. In the network plot, a "node" signifies an ingredient, target or pathway, an "edge" represents interaction among different targets. The "degree" of a node was in agreement with the number of its connected edges.

\subsection{Molecular docking and dynamics}

Surflex-Docking program in Sybyl X2.0 was utilized to evaluate the binding energy and interaction between key active compounds and targets. The crystallographic structures of 16 target proteins were retrieved from the RCSB Protein Data Bank database (http://www.rcsb.org). The binding energy could be accomplished by the formation of binding pockets after preparation of ligands and receptors by removal of water molecules and original ligands, addition of hydrogen atoms and repairmen of amino acids. "Total Score" was used as the indicator and positive correlation with docking preference. Score $\geq 4$ was considered meaningful, which mean that there was a binding between constituents and targets.

\subsection{Experimental validation in rat with $A L D$}

SD rats were randomly divided into 4 groups, including control group, alcoholic liver injury (ALD) model group, PF treatment $\left(1.1 \mathrm{~g} \mathrm{~kg}^{-1}\right)$ group, and tiopronin treatment $(60 \mathrm{mg}$ 
$\mathrm{kg}^{-1}$ ) group ( $\mathrm{n}=10$ in each group). The medicine-treated rats were pretreated with PF extract or tiopronin by intragastric administration twice daily for 1 week before the first dose of ethanol and at $1 \mathrm{~h}$ before each administration of ethanol doses for 4 weeks, whereas the rats in the control and model group were given equivalent volume of $0.5 \%$ sodium carboxymethylcellulose. Except for the control group, the ALD model was simultaneously induced by orally feeding $56 \%$ Er-Guo-Tou wine $(10 \mathrm{~mL} / \mathrm{kg} / \mathrm{d})$ by gavage for 4 weeks. $12 \mathrm{~h}$ after the final administration, blood was collected and centrifuged at $3000 \mathrm{r} / \mathrm{min}$ for $15 \mathrm{~min}$ to obtain serum. Liver tissues were harvested and divided into two parts, one was fixed in $4 \%$ paraformaldehyde for histological observation, another was immediately stored in $-80 \circ \mathrm{C}$ for the subsequent protein validation experiments.

The levels of alanine transaminase (ALT), aspartate transaminase (AST) and alkaline phosphatase (ALP) in serum were measured with the automatic biochemistry analyzer (Hitachi, 7600-020, Tokyo, Japan). The middle lobe of liver tissues was collected, sectioned and fixed in 4\% paraformaldehyde for at least $24 \mathrm{~h}$. After dehydrated in ethanol and embedded in paraffin, a series of paraffin sections $(5 \mu \mathrm{m})$ were stained with hematoxylin-eosin (H\&E) for histopathological examination.

Liver tissues were lysed with lysis buffer containing 1\% PMSF, phosphatase inhibitors and protease inhibitors, and incubated in an ice bath for $30 \mathrm{~min}$ to extract total protein. The concentration of the extracted protein was measured by BCA quantitative method. Equal amount of protein was electrophoretically separated by 10\% SDS-PAGE. After electrophoresis, the protein was transferred using a PVDF and then blocked in 5\% milk with TBST for 2 hours. The membranes were immersed in respective primary antibody of PPAR $\alpha$ (1:1000 dilution), MAO-A (1:1000 dilution) and $\beta$-actin (1:50000 dilution) at $4{ }^{\circ} \mathrm{C}$ overnight, and the next day incubated with horseradish peroxidase-conjugated secondary antibody at room temperature for 2h. Protein bands were detected with ECL Plus chemiluminescence reagent and quantified using Image $\mathbf{J}$ software (National Institutes of Health, USA). The values for each target protein were normalized to $\beta$-actin.

\subsection{Statistical analysis}

All values were expressed as mean \pm standard deviation (SD). Differences between different groups were analyzed with one-way analysis of variance (ANOVA) using GraphPad 
Prism 8.0.1. The value $p<0.05$ was considered as statistically significant.

239

240

\section{Results}

\subsection{Identification of absorbed constituents in vivo}

In order to identify the absorbed constituents and in vivo metabolites in rats, a total of 43 chemical constituents, including 22 isoflavonoids, 14 saponins, 6 flavonoids and 1 phenolic acid were identified or tentatively characterized in PF extracts by UPLC-QTOF-MS based on our previous literatures [5] (Supplemental Figure 1, Supplemental Table 1). With the help of employing the Metabolynx ${ }^{\mathrm{TM}}$ software combined with mass defect filtering (MDF) technique, the identification of potential metabolites could be obtained by comparing the extraction ion chromatograms of rat dosed biosamples with control biosamples (Figure 2).

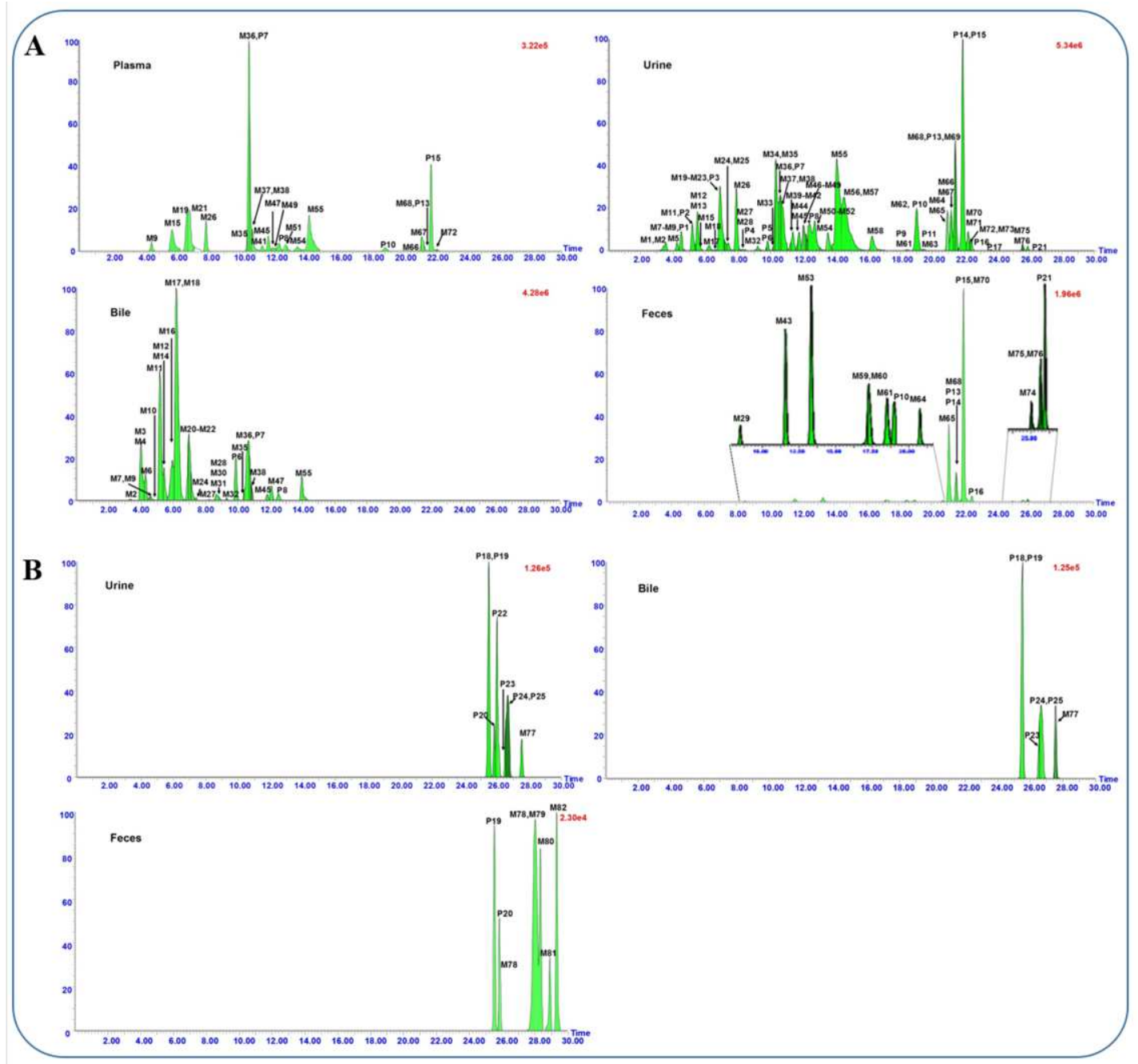

Figure 2 UPLC-QTOF/MS extracted ion chromatograms of flavonoids (A) and saponins (B) related metabolites in rat plasma, urine, bile and feces samples after oral administration of the flower of Pueraria thomsonii Benth extract. 
In total, 25 prototype components and 82 metabolites, including 93 flavonoids, 13 saponins and 1 phenolic acid were detected in vivo. In addition, the peak area of each absorbed constituent was also recorded automatically using the MetaboLynx ${ }^{\mathrm{TM}}$ system. After summarizing the peak area of all constituents, the percentage that calculated by the ratio of their peak area to total peak area in each biological sample was described as relative content. The detail data of identified results were listed in Table 1. These compounds could be generally divided into two categories, namely flavonoids-related and saponins-related metabolites.

\subsubsection{Metabolites associated with flavonoids}

A total of 93 flavonoids and their metabolites were screened out from the dosed samples, with 16 of them definitely elucidated as prototypes and others assigned as metabolites. Among the prototypes, 8 components that were almost consistent with our previous study, belong to aglycones (P9 P11, P13 P17 and P21) and glycosides (P1 P8), respectively. 5 (P7 P8, P10, P13, P15), 25 (P1 P25), 3 (P6 P8) and 6 (P10, P13 P16, P21) prototype components were observed separately in rat plasma, urine, bile and feces samples. As for the metabolites, glucuronidation, sulfation, methylation, hydroxylation and reduction were their major metabolic pathways. 76 metabolites, including 24 sulfates, 21 glucuronides, 17 aglycones, 8 glucuronide-sulfates, 4 diglucuronides and 2 disulfates were identified (Figure 2A).

As for aglycone skeleton, $\mathrm{MS}^{2}$ spectra with high energy showed characteristic ${ }^{1,3} \mathrm{~A}^{-}$and ${ }^{1,3} \mathrm{~B}^{-}$ions origin from a retro-Diels-Alder (RDA) cleavage of $\mathrm{C}$ ring as well as losses of $\mathrm{CH}_{3}$ (15 Da), O (16 Da), $\mathrm{H}_{2} \mathrm{O}(18 \mathrm{Da}), \mathrm{CO}(28 \mathrm{Da}), \mathrm{CO}_{2}(44 \mathrm{Da})$ and/or combination of the fragments above-mentioned. Meanwhile, the reduction at 2,3-double bond of C-ring and rearrangement between C-6 and C-8 positions were also common in vivo metabolic pathway of PF flavonoids according to related literature (Bai et al., 2010; Bai et al., 2011a). Correspondingly, metabolic pathway of flavonoids from PF in vivo was outlined as shown in Figure 3. The results indicated that irilin D (P9), glycitein (P10), genistein (P13), tectorigenin (P15), iristectorigenin A (P16) and irisolidone (P21), which were the aglycone of flavonoids constituent in PF extracts, were key prototype components in the metabolic process. 


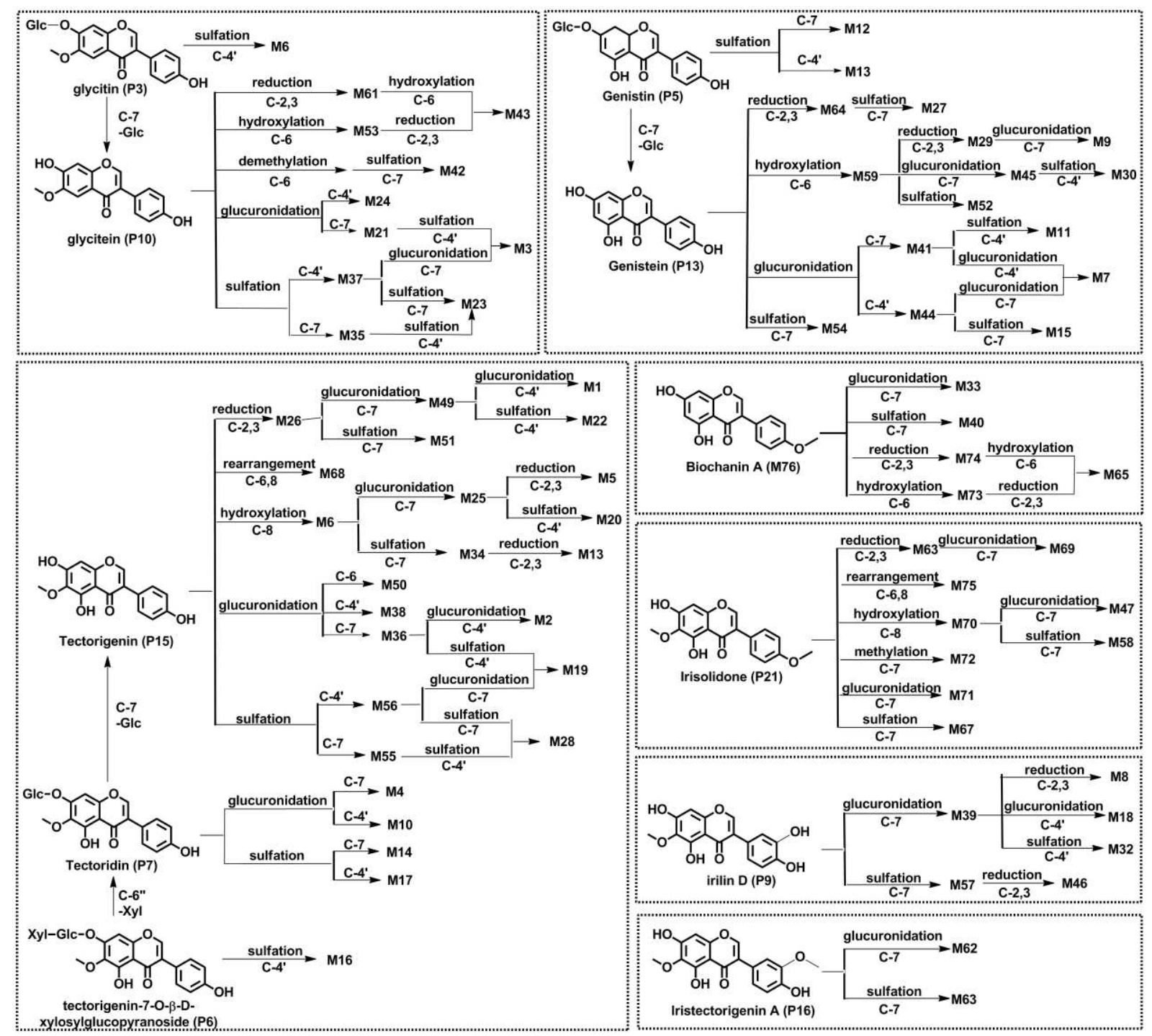

280 Figure 3 Proposed metabolic pathways of flavonoids related metabolites in rats after oral administration of the flower of Pueraria thomsonii Benth extract.

\subsubsection{Metabolites associated with saponins}

Triterpenoid saponins found in PF are another major group of bioactive components. 13 saponins, shared a basic chemical skeleton but with different moieties at C-3, C-22 and C-28, were identified and tentatively characterized based on their mass spectra and literature reports [16-18] (Figure 2B).

Compounds P18 P20, M78 and M81 M82 could be grouped due to possessing the same aglycone soyasapogenol $\mathrm{B}\left(\mathrm{C}_{30} \mathrm{H}_{50} \mathrm{O}_{3}\right)$ as the aglycone, and they presented similar fragmentation pathways. P18 and P20 with precursor ions detected at $m / z 911[\mathrm{M}-\mathrm{H}]^{-}$are a pair of isomeric with the identical elemental composition of $\mathrm{C}_{47} \mathrm{H}_{76} \mathrm{O}_{17}$. Both displayed a series of 
characteristic fragment ions at $m / z 765,615,457$ and 437 by simultaneously losing sugar units including $146 \mathrm{Da}$ (rhamonose), $132 \mathrm{Da}$ (arabinose, xylose), $176 \mathrm{Da}$ (glucuronosyl) and $18 \mathrm{Da}$ $\left(\mathrm{H}_{2} \mathrm{O}\right)$ at the site of C-3. They were ascribed to astragaloside VIII and soyasaponin II, respectively [5]. Similarly, P19 was identified as soyasaponin I according to our previous reports [5]. The metabolites M78 and M81 exhibited the protonated molecular ion at $\mathrm{m} / \mathrm{z}$ $635.4177\left(\mathrm{C}_{36} \mathrm{H}_{60} \mathrm{O} 9\right)$ and $m / z, 649.4307\left(\mathrm{C}_{37} \mathrm{H}_{62} \mathrm{O} 9\right)$, which was $2 \mathrm{Da}(2 \mathrm{H})$ and $16 \mathrm{Da}\left(\mathrm{CH}_{2}+2 \mathrm{H}\right)$ higher than protonated ion of soyasapogenol B-3- $\beta$-D-glucuronide, suggesting that both were reduced and methylated metabolites. Reduction at 12,13-double bond of C-ring is a metabolic pathway for saponins such as $\alpha$-hederin [17], which were also reduced to hydrogenated metabolite in rat feces by the gut microflora. Accordingly, M78 and M81 was tentatively identified as reduct-soyasapogenol B-3- $\beta$-D-glucuronide and methyl-reduct-soyasapogenol B3- $\beta$-D-glucuronide based on the above researches. Similarly, M82 with $m / z 473.3635\left(\mathrm{C}_{30} \mathrm{H}_{50} \mathrm{O}_{4}\right)$ was $16 \mathrm{Da}(\mathrm{O})$ higher than that of aglycone, which was identified as 1-hydroxyl-soyasapogenol $\mathrm{B}$ as $\mathrm{C}-1$ was the active site according to related research [18].

P22 P25 and M77 could be grouped due to possessing the same aglycone sophoradiol. P22 P25 were identified as kaikasaponin III, kaikasaponin I, kakkasaponin I and baptisiasaponin I by comparion with the identified constituent in PF extracts, respectively [5]. M77 exhibited a protonated molecular ion at $\mathrm{m} / \mathrm{z} 749.4461\left(\mathrm{C}_{41} \mathrm{H}_{66} \mathrm{O}_{12}\right)$, which was $30 \mathrm{Da}(-$ $\mathrm{CH}_{2}-\mathrm{O}$ ) lower than the protonated ion of kakkasaponin I (P23). Thus, it was demethylated and dehydroxylated metabolite of P23. Since there is only one hydroxyl substituted at C-22, M77 was deduced as dimethyl-22-dehydroxyl-kakkasaponin I. Analogously, M79 and M80 was identified as methylated and C1, C21-dihydroxyl of aglycone soyasapogenol E based on similar metabolic pathway of saikosaponin $\mathrm{G}$ and glycyrrhetinic acid $[16,18]$. 
Table 1 Characterization of the metabolites in rat after oral administration of Pueraria Flower by UHPLC-QTOF-MS

\begin{tabular}{|c|c|c|c|c|c|c|c|c|c|c|c|}
\hline \multirow[t]{2}{*}{ No. } & \multirow{2}{*}{$\begin{array}{c}\mathrm{t}_{\mathrm{R}} \\
(\mathrm{min})\end{array}$} & \multirow[t]{2}{*}{ Formula } & \multicolumn{4}{|c|}{$[\mathrm{M}-\mathrm{H}]^{-}$} & \multirow[t]{2}{*}{ Identification } & \multicolumn{4}{|c|}{${\text { Relative content }(\%)^{a}}^{a}$} \\
\hline & & & Calculated & Observed & $\mathrm{ppm}$ & MS/MS fragments & & $\mathrm{P}$ & $\mathrm{U}$ & $\mathrm{B}$ & $\mathrm{F}$ \\
\hline M1 & 3.26 & $\mathrm{C}_{28} \mathrm{H}_{30} \mathrm{O}_{18}$ & 653.1354 & 653.1315 & -5.97 & $477,301,286$ & dihydrotectorigenin-7G-4'G & - & 0.03 & - & - \\
\hline M2 & 3.53 & $\mathrm{C}_{28} \mathrm{H}_{28} \mathrm{O}_{18}$ & 651.1197 & 651.1194 & -0.46 & $475,299,284$ & tectorigenin-7G-4'G & - & 0.50 & 0.16 & - \\
\hline M3 & 3.98 & $\mathrm{C}_{22} \mathrm{H}_{20} \mathrm{O}_{14} \mathrm{~S}$ & 539.0496 & 539.0497 & 0.19 & $363,283,268$ & glycitein-7G-4'-S & - & - & 5.90 & - \\
\hline M4 & 4.1 & $\mathrm{C}_{28} \mathrm{H}_{30} \mathrm{O}_{17}$ & 637.1405 & 637.1406 & 0.16 & $461,299,284$ & tectoridin-7G & - & - & 0.84 & - \\
\hline M5 & 4.2 & $\mathrm{C}_{22} \mathrm{H}_{22} \mathrm{O}_{13}$ & 493.0982 & 493.097 & -2.43 & $317,301,286$ & 8-OH-dihydrotectorigenin-7G & - & 0.34 & - & 一 \\
\hline M7 & 4.44 & $\mathrm{C}_{27} \mathrm{H}_{26} \mathrm{O}_{17}$ & 621.1092 & 621.1105 & 2.09 & $445,269,133$ & genistein-7G-4'G & - & 0.17 & 0.18 & 一 \\
\hline M8 & 4.45 & $\mathrm{C}_{22} \mathrm{H}_{22} \mathrm{O}_{13}$ & 493.0982 & 493.0974 & -1.62 & 317,302 & dihydro-irilinD-7G & - & 0.29 & - & 一 \\
\hline M9 & 4.51 & $\mathrm{C}_{21} \mathrm{H}_{20} \mathrm{O}_{12}$ & 463.0877 & 463.0861 & -3.46 & 287,259 & 6-OH-dihydrogenistein -7G & 1.18 & 0.44 & 0.28 & - \\
\hline P1 & 4.69 & $\mathrm{C}_{21} \mathrm{H}_{22} \mathrm{O}_{11}$ & 449.1084 & 449.1083 & -0.22 & 287,269 & dihydrokaempferol-7-O-glucoside & - & 0.03 & - & - \\
\hline M10 & 4.76 & $\mathrm{C}_{28} \mathrm{H}_{30} \mathrm{O}_{17}$ & 637.1405 & 637.1455 & 7.85 & $461,299,284$ & tectoridin-4'G & - & - & 0.06 & 一 \\
\hline M11 & 5.16 & $\mathrm{C}_{21} \mathrm{H}_{18} \mathrm{O}_{14} \mathrm{~S}$ & 525.0339 & 525.0337 & -0.38 & $349,269,133$ & genistein-7G-4'S & - & 1.27 & 15.57 & - \\
\hline M12 & 5.38 & $\mathrm{C}_{21} \mathrm{H}_{20} \mathrm{O}_{13} \mathrm{~S}$ & 511.0546 & 511.0534 & -2.35 & $431,269,133$ & genistin-7S & - & 0.08 & 2.36 & - \\
\hline M13 & 5.48 & $\mathrm{C}_{16} \mathrm{H}_{14} \mathrm{O}_{10} \mathrm{~S}$ & 397.0229 & 397.0228 & -0.25 & $317,301,286$ & 8-OH-dihydrotectorigenin-7S & - & 1.86 & - & 一 \\
\hline M14 & 5.53 & $\mathrm{C}_{22} \mathrm{H}_{22} \mathrm{O}_{14} \mathrm{~S}$ & 541.0652 & 541.0621 & -5.73 & $461,299,284$ & tectoridin-7S & - & - & 0.26 & 一 \\
\hline M15 & 5.79 & $\mathrm{C}_{21} \mathrm{H}_{18} \mathrm{O}_{14} \mathrm{~S}$ & 525.0339 & 525.0361 & 4.19 & $349,269,133$ & genistein-7S-4'G & 6.05 & 0.02 & - & 一 \\
\hline M16 & 5.92 & $\mathrm{C}_{27} \mathrm{H}_{30} \mathrm{O}_{18} \mathrm{~S}$ & 673.1075 & 673.1075 & 0.00 & $379,299,284$ & Te-7XG-4'S & - & - & 6.52 & 一 \\
\hline M17 & 6.21 & $\mathrm{C}_{22} \mathrm{H}_{22} \mathrm{O}_{14} \mathrm{~S}$ & 541.0652 & 541.0643 & -1.66 & $461,299,284$ & TE-4'S & - & 0.34 & 31.08 & - \\
\hline M18 & 6.56 & $\mathrm{C}_{28} \mathrm{H}_{28} \mathrm{O}_{19}$ & 667.1147 & 667.1157 & 1.50 & $491,315,300$ & irilin D-7G-4'G & - & 0.04 & 0.04 & - \\
\hline M19 & 6.71 & $\mathrm{C}_{22} \mathrm{H}_{20} \mathrm{O}_{15} \mathrm{~S}$ & 555.0445 & 555.0441 & -0.72 & $379,299,284$ & Te-7G-4'S & 12.17 & 22.71 & - & - \\
\hline M20 & 6.87 & $\mathrm{C}_{22} \mathrm{H}_{20} \mathrm{O}_{16} \mathrm{~S}$ & 571.0394 & 571.0394 & 0.00 & $395,315,299,284$ & 8-OH-Te-7G-4'S & - & 2.48 & 4.65 & - \\
\hline M21 & 6.89 & $\mathrm{C}_{22} \mathrm{H}_{20} \mathrm{O}_{11}$ & 459.0927 & 459.0915 & -2.61 & 283,268 & glycitein-7G & 3.61 & 2.03 & 4.49 & - \\
\hline M22 & 7.14 & $\mathrm{C}_{22} \mathrm{H}_{22} \mathrm{O}_{15} \mathrm{~S}$ & 557.0601 & 557.0587 & -2.51 & $477,301,286$ & dihydrotectorigenine-7G-4'S & - & 0.35 & 0.83 & - \\
\hline M23 & 7.17 & $\mathrm{C}_{16} \mathrm{H}_{12} \mathrm{O}_{11} \mathrm{~S}_{2}$ & 442.9743 & 442.9748 & 1.13 & $363,283,268$ & glycitein-7S-4'S & - & 0.02 & - & 一 \\
\hline P3 & 7.28 & $\mathrm{C}_{22} \mathrm{H}_{22} \mathrm{O}_{10}$ & 445.1135 & 445.1136 & 0.22 & $283,268,239$ & glycitin & - & 0.04 & - & - \\
\hline
\end{tabular}


Table 1 Characterization of the metabolites in rat after oral administration of Pueraria Flower by UHPLC-QTOF-MS (Continued)

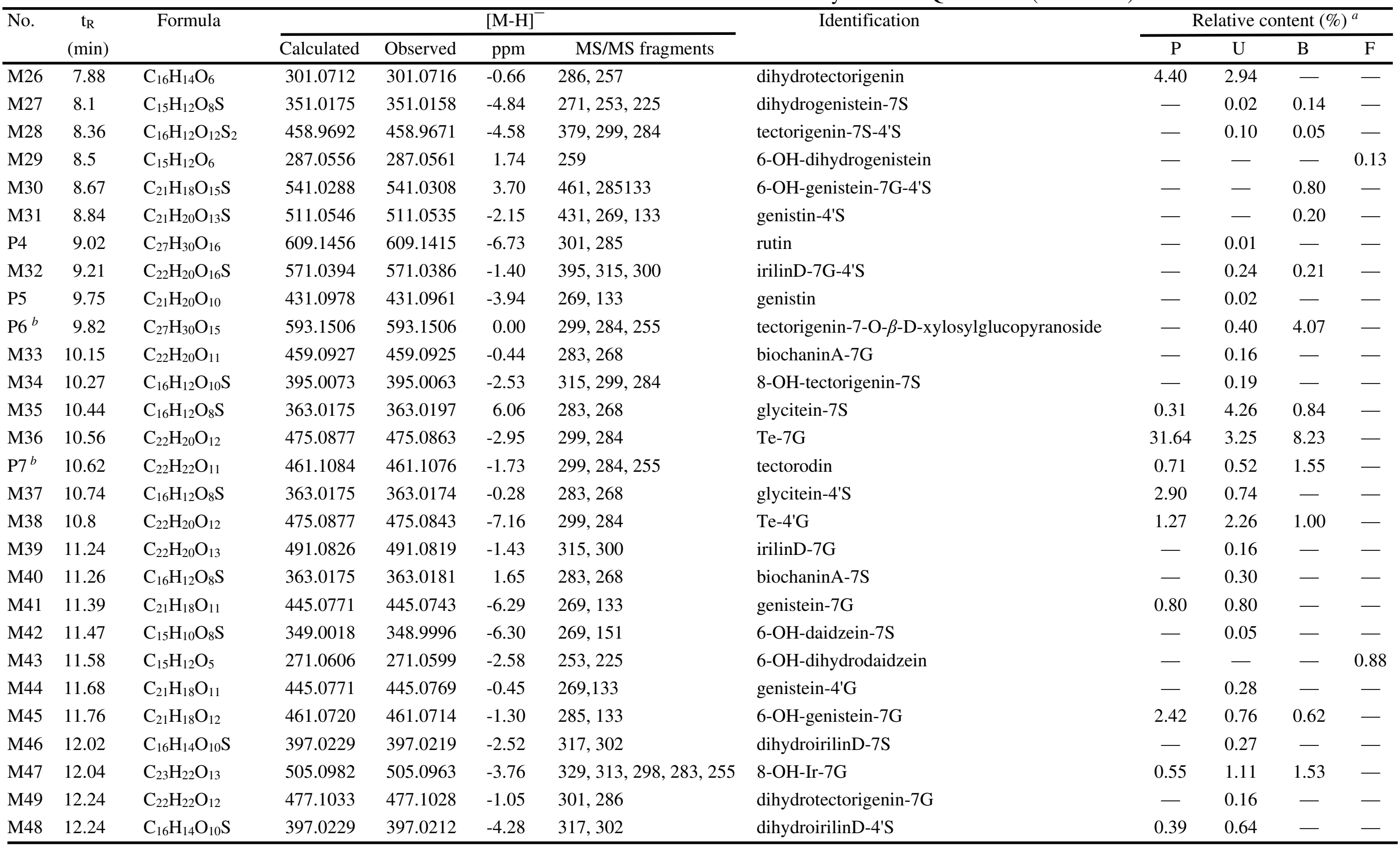


Table 1 Characterization of the metabolites in rat after oral administration of Pueraria Flower by UHPLC-QTOF-MS (Continued)

\begin{tabular}{|c|c|c|c|c|c|c|c|c|c|c|c|}
\hline \multirow[t]{2}{*}{ No. } & \multirow{2}{*}{$\begin{array}{c}\mathrm{t}_{\mathrm{R}} \\
(\mathrm{min})\end{array}$} & \multirow[t]{2}{*}{ Formula } & \multicolumn{4}{|c|}{$[\mathrm{M}-\mathrm{H}]^{-}$} & \multirow[t]{2}{*}{ Identification } & \multicolumn{4}{|c|}{ Relative content $(\%)^{a}$} \\
\hline & & & Calculated & Observed & ppm & MS/MS fragments & & $\mathrm{P}$ & $\mathrm{U}$ & $\mathrm{B}$ & $\mathrm{F}$ \\
\hline P8 & 12.43 & $\mathrm{C}_{21} \mathrm{H}_{20} \mathrm{O}_{11}$ & 447.0927 & 447.0971 & 9.84 & 285,267 & 6-hydroxygenistein-7-O-glucoside & 1.56 & 0.28 & 0.84 & - \\
\hline M50 & 12.75 & $\mathrm{C}_{22} \mathrm{H}_{20} \mathrm{O}_{12}$ & 475.0877 & 475.0872 & -1.05 & $299,284,255$ & 6-OH-biochanin A-6G & - & 1.25 & - & - \\
\hline M51 & 12.86 & $\mathrm{C}_{16} \mathrm{H}_{14} \mathrm{O}_{9} \mathrm{~S}$ & 381.028 & 381.0269 & -2.89 & 301,286 & dihydrotectorigenin-7S & 1.51 & 2.50 & - & - \\
\hline M52 & 13.03 & $\mathrm{C}_{15} \mathrm{H}_{10} \mathrm{O}_{9} \mathrm{~S}$ & 364.9967 & 364.9967 & 0.00 & 285,257 & 6-OH-genistein-7S & - & 0.19 & - & - \\
\hline M53 & 13.34 & $\mathrm{C}_{15} \mathrm{H}_{10} \mathrm{O}_{5}$ & 269.045 & 269.0448 & -0.74 & 151 & 6-OH-daidzein & - & - & - & 1.35 \\
\hline M55 & 14.2 & $\mathrm{C}_{16} \mathrm{H}_{12} \mathrm{O}_{9} \mathrm{~S}$ & 379.0124 & 379.0116 & -2.11 & 299,284 & $\mathrm{Te}-7 \mathrm{~S}$ & 12.10 & 8.75 & 2.64 & - \\
\hline M56 & 14.53 & $\mathrm{C}_{16} \mathrm{H}_{12} \mathrm{O}_{9} \mathrm{~S}$ & 379.0124 & 379.0133 & 2.37 & 299,284 & Te-4'S & - & 6.97 & - & - \\
\hline M57 & 15.01 & $\mathrm{C}_{16} \mathrm{H}_{12} \mathrm{O}_{10} \mathrm{~S}$ & 395.0073 & 395.0068 & -1.27 & 315,300 & irilinD-7S & - & 1.00 & - & - \\
\hline M58 & 16.23 & $\mathrm{C}_{17} \mathrm{H}_{14} \mathrm{O}_{10} \mathrm{~S}$ & 409.0229 & 409.0227 & -0.49 & $329,313,298,283$ & 8-OH-Ir-7S & - & 1.26 & - & - \\
\hline M59 & 17.19 & $\mathrm{C}_{15} \mathrm{H}_{10} \mathrm{O}_{6}$ & 285.0399 & 285.0392 & -2.46 & 257,229 & 6-OH-genistein & - & - & - & 0.26 \\
\hline M60 & 17.26 & $\mathrm{C}_{16} \mathrm{H}_{12} \mathrm{O}_{7}$ & 315.0505 & 315.0511 & 1.90 & $299,284,255$ & 8-OH-tectorigenin & - & - & - & 0.47 \\
\hline M61 & 18.49 & $\mathrm{C}_{16} \mathrm{H}_{14} \mathrm{O}_{5}$ & 285.0763 & 285.076 & -1.05 & 270 & dihydroglycitein & - & 0.03 & - & 0.54 \\
\hline P10 & 19.02 & $\mathrm{C}_{16} \mathrm{H}_{12} \mathrm{O}_{5}$ & 283.0606 & 283.0614 & 2.83 & 268 & glycitein & 0.68 & 2.62 & - & 0.45 \\
\hline M62 & 19.02 & $\mathrm{C}_{23} \mathrm{H}_{22} \mathrm{O}_{13}$ & 505.0982 & 505.0937 & -8.91 & 329,314 & iristectorigeninA-7G & - & 0.27 & - & - \\
\hline $\mathrm{P} 11^{b}$ & 19.47 & $\mathrm{C}_{15} \mathrm{H}_{10} \mathrm{O}_{6}$ & 285.0399 & 285.0382 & -5.96 & 133 & luteolin & - & 0.09 & - & - \\
\hline M63 & 19.86 & $\mathrm{C}_{17} \mathrm{H}_{16} \mathrm{O}_{6}$ & 315.0869 & 315.0845 & -7.62 & $300,285,257$ & dihydroirisolidone & - & 0.01 & - & - \\
\hline P12 & 20.13 & $\mathrm{C}_{8} \mathrm{H}_{8} \mathrm{O}_{4}$ & 167.0344 & 167.0345 & 0.60 & 108 & vanillic acid & 0.51 & 0.04 & - & - \\
\hline M64 & 20.72 & $\mathrm{C}_{15} \mathrm{H}_{12} \mathrm{O}_{5}$ & 271.0606 & 271.0589 & -6.27 & 253,225 & dihydrogenistein & - & 0.14 & - & 0.26 \\
\hline M65 & 20.92 & $\mathrm{C}_{16} \mathrm{H}_{14} \mathrm{O}_{6}$ & 301.0712 & 301.0711 & -0.66 & 286,257 & 6-OH-dihydrobiochaninA & - & 1.67 & - & 21.96 \\
\hline M66 & 21.14 & $\mathrm{C}_{17} \mathrm{H}_{14} \mathrm{O}_{10} \mathrm{~S}$ & 409.0229 & 409.0215 & -3.42 & 329,314 & iristectorigeninA-7S & 0.43 & 0.54 & - & - \\
\hline M67 & 21.3 & $\mathrm{C}_{17} \mathrm{H}_{14} \mathrm{O}_{9} \mathrm{~S}$ & 393.028 & 393.0269 & -2.80 & $313,298,283,255$ & Ir-7S & 1.85 & 1.42 & - & - \\
\hline M68 & 21.41 & $\mathrm{C}_{16} \mathrm{H}_{12} \mathrm{O}_{6}$ & 299.0556 & 299.0553 & -1.00 & 284,255 & isotectorigenin & 0.25 & 1.80 & - & 5.69 \\
\hline $\mathrm{P} 13^{b}$ & 21.43 & $\mathrm{C}_{15} \mathrm{H}_{10} \mathrm{O}_{5}$ & 269.045 & 269.0451 & 0.37 & 133 & genistein & 0.59 & 3.02 & - & 2.74 \\
\hline M69 & 21.59 & $\mathrm{C}_{23} \mathrm{H}_{24} \mathrm{O}_{12}$ & 491.119 & 491.1167 & -4.68 & $315,300,285$ & dihydroirisolidone-7G & - & 0.03 & - & - \\
\hline
\end{tabular}


Table 1 Characterization of the metabolites in rat after oral administration of Pueraria Flower by UHPLC-QTOF-MS (Continued)

\begin{tabular}{|c|c|c|c|c|c|c|c|c|c|c|c|}
\hline \multirow[t]{2}{*}{ No. } & \multirow{2}{*}{$\begin{array}{c}\mathrm{t}_{\mathrm{R}} \\
(\mathrm{min})\end{array}$} & \multirow[t]{2}{*}{ Formula } & \multicolumn{4}{|c|}{$[\mathrm{M}-\mathrm{H}]^{-}$} & \multirow[t]{2}{*}{ Identification } & \multicolumn{4}{|c|}{ Relative content $(\%)^{a}$} \\
\hline & & & Calculated & Observed & ppm & MS/MS fragments & & $\mathrm{P}$ & $\mathrm{U}$ & $\mathrm{B}$ & $\mathrm{F}$ \\
\hline M70 & 22.13 & $\mathrm{C}_{17} \mathrm{H}_{14} \mathrm{O}_{7}$ & 329.0661 & 329.0678 & 5.17 & $313,298,283,255$ & 8-OH-Ir & - & 0.05 & - & 0.21 \\
\hline M71 & 22.2 & $\mathrm{C}_{23} \mathrm{H}_{22} \mathrm{O}_{12}$ & 489.1033 & 489.1028 & -1.02 & $313,298,283,255$ & Ir-7G & - & 0.68 & - & - \\
\hline M72 & 22.24 & $\mathrm{C}_{18} \mathrm{H}_{16} \mathrm{O}_{6}$ & 327.0869 & 327.0883 & 4.28 & $313,298,283,255$ & 4',7-di-methyltectorigenin & 0.14 & 0.01 & - & - \\
\hline M73 & 22.3 & $\mathrm{C}_{16} \mathrm{H}_{12} \mathrm{O}_{6}$ & 299.0556 & 299.0554 & -0.67 & 284,255 & 6-OH-biochaninA & - & 0.35 & - & - \\
\hline P16 & 22.42 & $\mathrm{C}_{17} \mathrm{H}_{14} \mathrm{O}_{7}$ & 329.0661 & 329.066 & -0.30 & 314 & iristectorigenin $\mathrm{A}$ & - & 0.24 & - & 1.55 \\
\hline M74 & 25.03 & $\mathrm{C}_{16} \mathrm{H}_{14} \mathrm{O}_{5}$ & 285.0763 & 285.0751 & -4.21 & 270 & dihydrobiochanin $\mathrm{A}$ & - & - & - & 0.13 \\
\hline P18 & 25.47 & $\mathrm{C}_{47} \mathrm{H}_{76} \mathrm{O}_{17}$ & 911.5004 & 911.4987 & -1.87 & $765,615,457,437$ & astragaloside VIII & - & 0.04 & 0.14 & - \\
\hline P19 & 25.52 & $\mathrm{C}_{48} \mathrm{H}_{78} \mathrm{O}_{18}$ & 941.511 & 941.5088 & -2.34 & $795,615,457,437$ & soyasaponin I & - & 0.15 & 0.36 & 0.76 \\
\hline M75 & 25.56 & $\mathrm{C}_{17} \mathrm{H}_{14} \mathrm{O}_{6}$ & 313.0712 & 313.072 & -0.66 & $298,283,255$ & isoirisolidone & - & 0.04 & - & 0.13 \\
\hline M76 & 25.69 & $\mathrm{C}_{16} \mathrm{H}_{12} \mathrm{O}_{5}$ & 283.0606 & 283.0605 & -0.35 & 268 & biochanin $\mathrm{A}$ & - & 0.21 & - & 0.25 \\
\hline $\mathrm{P} 20$ & 25.86 & $\mathrm{C}_{47} \mathrm{H}_{76} \mathrm{O}_{17}$ & 911.5004 & 911.5 & -0.44 & $765,615,457,437$ & soyasaponin II & - & 0.04 & - & 0.29 \\
\hline $\mathrm{P} 22$ & 26.01 & $\mathrm{C}_{48} \mathrm{H}_{78} \mathrm{O}_{17}$ & 925.5161 & 925.517 & 0.97 & $779,599,441,439$ & kaikasaponin III & - & 0.16 & - & - \\
\hline $\mathrm{P} 23$ & 26.57 & $\mathrm{C}_{42} \mathrm{H}_{68} \mathrm{O}_{13}$ & 779.4582 & 779.4576 & -0.77 & 617,439 & kaikasaponin I & - & 0.05 & 0.13 & - \\
\hline P24 & 26.66 & $\mathrm{C}_{47} \mathrm{H}_{76} \mathrm{O}_{16}$ & 895.5055 & 895.5039 & -1.79 & $599,441,439$ & kakkasapnin I & - & 0.05 & 0.11 & 一 \\
\hline $\mathrm{P} 25$ & 26.75 & $\mathrm{C}_{47} \mathrm{H}_{76} \mathrm{O}_{16}$ & 895.5055 & 895.5021 & -3.80 & $599,441,439$ & baptisiasaponin I & - & 0.05 & 0.10 & - \\
\hline M77 & 27.54 & $\mathrm{C}_{41} \mathrm{H}_{66} \mathrm{O}_{12}$ & 749.4476 & 749.4461 & -2.00 & $587,411,409$ & demethyl-22-dehydroxyl- kaikasaponin I & - & 0.03 & 0.16 & - \\
\hline M78 & 27.92 & $\mathrm{C}_{36} \mathrm{H}_{60} \mathrm{O}_{9}$ & 635.4159 & 635.4177 & 2.83 & 459,438 & reduct-soyasapogenol B-3- $\beta$-D-glucuronide & - & - & - & 0.14 \\
\hline M79 & 28.03 & $\mathrm{C}_{31} \mathrm{H}_{50} \mathrm{O}_{3}$ & 469.3682 & 469.3668 & -2.98 & $455,439,437$ & methyl- soyasapogenol E & - & - & - & 1.48 \\
\hline M80 & 28.32 & $\mathrm{C}_{30} \mathrm{H}_{48} \mathrm{O}_{5}$ & 487.3423 & 487.3398 & -5.13 & $471,455,439$ & 1, 21-dihydroxyl- soyasapogenol E & - & - & - & 0.55 \\
\hline M81 & 28.89 & $\mathrm{C}_{37} \mathrm{H}_{62} \mathrm{O}_{9}$ & 649.4316 & 649.4307 & -1.39 & $473,459,441,439$ & methyl-reduct-soyasapogenol B-3- $\beta$-D-glucuronide & - & - & - & 0.23 \\
\hline M82 & 29.31 & $\mathrm{C}_{30} \mathrm{H}_{50} \mathrm{O}_{4}$ & 473.3631 & 473.3635 & 0.85 & 457,437 & 1-hydroxyl-soyasapogenol B & - & - & - & 0.65 \\
\hline
\end{tabular}

${ }^{a} \mathrm{P}$, plasma samples; $\mathrm{U}$, urine samples; $\mathrm{B}$, bile samples; $\mathrm{F}$, fecal samples.

${ }^{b}$ Components identified with reference compounds comparison. 


\subsection{Compound-target-pathway network construction}

In order to understand important effective components, the relative content of each metabolite was calculated by area normalization and expressed as the percentage of its peak area to the total peak areas in each kind of bio-samples (Table 1). 13 candidate components that were detected in rat plasma and/or bile samples with relative content more than $3 \%$ were screened for the further network pharmacology analysis. In all, 104 and 5338 related genes were obtained as the targets of compounds and disease after removing duplicates, respectively. Their detail information is shown in Supplementary Table S2 and S3. To acquire the candidate targets the effects of PF against ALD, 75 intersection targets from above constituent-targets and disease-targets were obtained by Cytoscape software. Then, protein-protein interaction (PPI) analysis aiming at more crucial targets was carried out on the intersection targets by using STRING database. Finally, 43 candidate targets with confidence scores greater than 0.9 were screened and subjected to the KEGG pathway enrichment for elucidate the molecular mechanisms. Accordingly, the absorbed component-target-pathway network diagram was constructed (Figure 4).

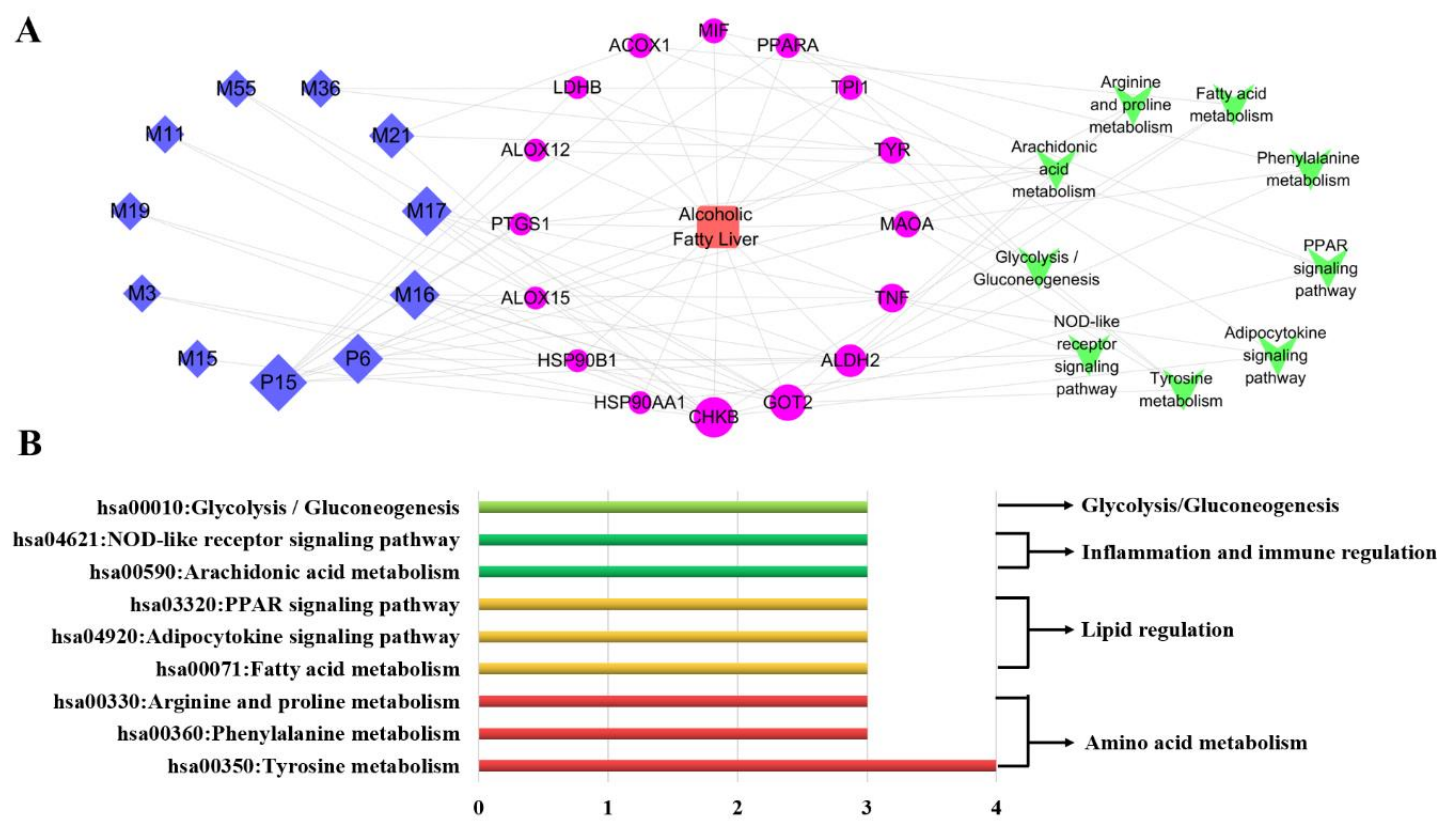

Figure 4. The interaction network of absorbed constituents, candidate targets and enriched pathways. A node stands for a constituent (blue square), a target (pink circle) or a pathway (green triangle), the interactions of two nodes were represented by a line and the bigger size of a node refers to a higher degree. 
As shown in Figure 4, eleven absorbed components (P6, P15, M3, M11, M15 M17, M19, M21, M36 and M55) were found to interact with the 16 relevant targets, which were sorted into four groups: (1) glycolysis/gluconeogenesis related targets (LDHB, TPI1 and ALDH2); (2) amino acid metabolism related targets (ALDH2, GOT2, MAOA, MIF and TYR); (3) lipid regulation related targets (ALDH2, ACOX1, CHKB, PPARA and TNF); (4) inflammation and immune regulation related targets (TNF, HSP90AA1, HSP90B1, ALOX12, ALOX15 and PTGS1). Among them, CHKB, GOT2 and ALDH2 were topologically important, and play a significant pharmacology role. Along similar lines, P15, P6 and M16 that possessed more interactions with targets may be the key components.

\subsection{Molecular docking}

Docking analysis was performed to evaluate the relationship between the active components and potential targets that were predicted by the network pharmacology. The aforementioned 11 absorbed ingredients were selected as candidate components to dock with the 16 relevant targets that were screened by the network pharmacology analysis. Consequently, a heatmap performed by Graphpad 8.0 software was present for intuitively describing the receptor-ligand interactions.

Based on the heatmap (Figure 5A), the interactions of 11 components with 16 selective targets were ranked as intensive binding (>8.0), moderate binding (6.0 8.0) and weak binding $(<6.0)$. Here, 8.0 was set as the cutoff value to screen the potential active components against AFL. As a result, Te-7XG (P6) and three metabolites genistein-7G-4'S (M11), tectoridin-4'S (M17), Te-7XG-4'S (M16) showed better binding ability with more than 3 intensive values, while weak or no inhibition effects were observed to the prototype Te (P15). As for targets, MAOA and PPARA that rank the top two places in binding ability, and that contained 7 and 6 values more than the cutoff, revealed close correlations with PF absorbed components. Among them, the interaction between MAOA and compounds M3, M11 and M16, and PPARA and M16 presented the best performance. P15 and M16 were selected as representative prototype components and metabolites, respectively. Their binding mode in the active site of MAOA and PPARA has been shown in a three-dimensional pattern in Figure 5B. 

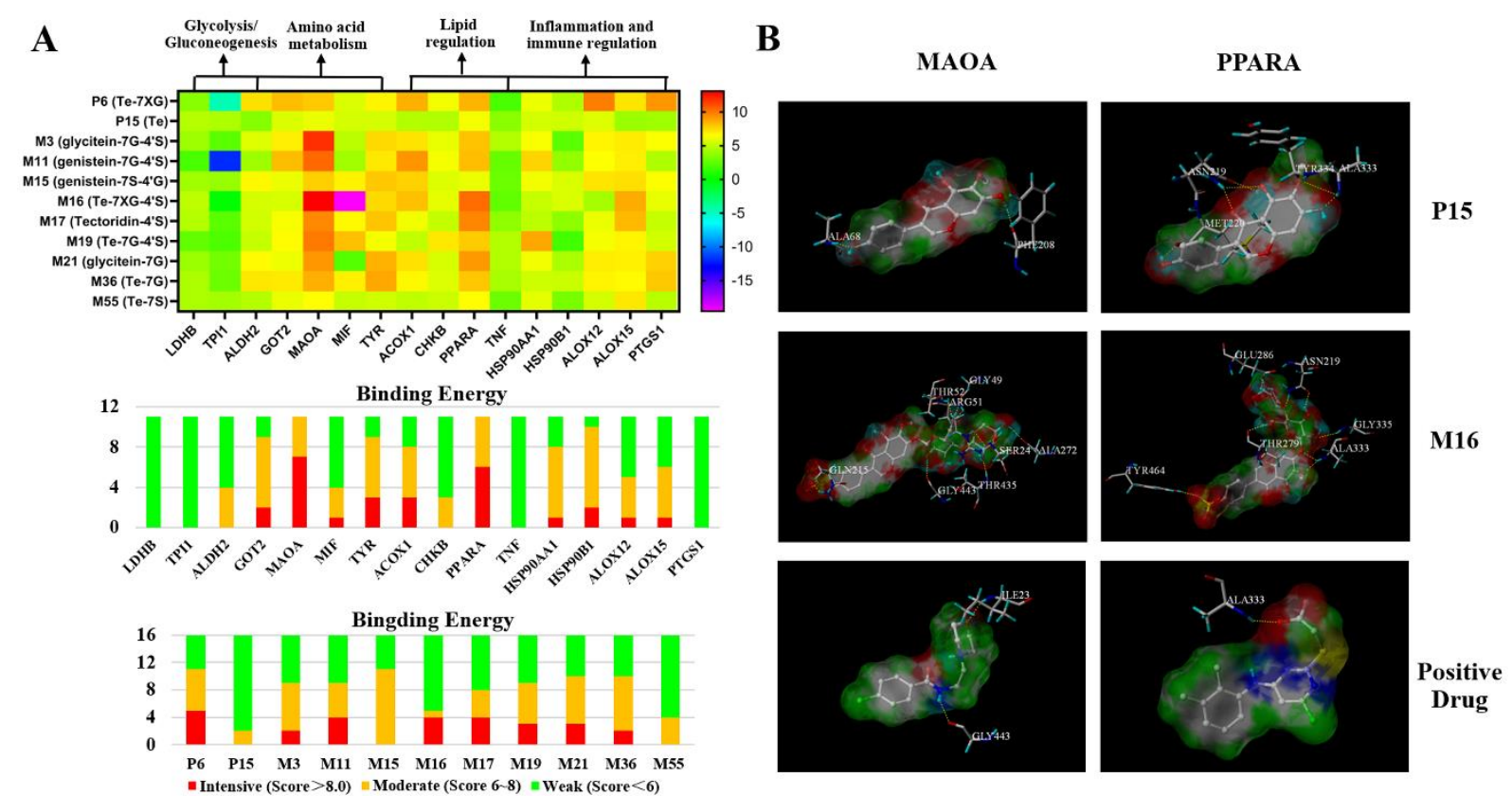

Figure 5. Binding interaction between the active metabolites and potential targets predicted by network pharmacology. (A) Binding energy between 11 constituents with 16 targets performed by docking. (B) The binding mode of Te (P15), Te-7XG-4'S (M16) and positive drug with MAOA and PPARA. (Compounds are presented with thick stick style; Hydrogen bonding interactions are expressed by the yellow dotted lines. Amino acid residues which form H-bonds are presented by thin line and labeled with residue sequence).

As for MAOA, P15 showed two H-bond interactions with PHE208 and ALA68, which were $\mathrm{C}-7$ and $\mathrm{C}-4$ ' phenolic hydroxyls on the A and B rings, respectively. M16 showed eight $\mathrm{H}-$ bond interactions as follows: four hydroxyl groups on glucose with Gly443, Gly49, ARG51 and THR52, three hydroxyl groups on xylose with THR435, SER24 and ALA27, and a phenolic hydroxyl at C-4' on the B ring with GLN215. While Gly443 and ILE23 were involved in the $\mathrm{N}-\mathrm{H}$ Bond interactions with a representative MAOA inhibitor moclobemide, the former was also the connection site of M16 with MAOA.

Along the similar lines, interaction between P15 and PPARA showed three H-bond interactions, a phenolic hydroxyl and a carbonyl group from the isoflavonoid skeleton with ASN 219, a methoxy group and a phenolic hydroxyl on the A ring with ALA333, and a methoxy group on the A ring with TYR334. Similarly, M16 showed five H-bond interactions, two phenolic hydroxyls with ASN219 and ALA333, and three hydroxyl groups on glucose and 
xylose with THR279, Glu286 and Gly335. Besides, the interaction of C-4'-sulfate with TYR 279 was also observed. As the positive comparison, PPARA agonist WY14643 shared the same residue ALA333 with P15 and M16, which was the active site for the treatment on ALD. Collectively, the docking results above showed that glycosylation or sulfation increased the binding activity comparison with the prototype.

\subsection{Experimental validations of the pharmacological effects and the molecular mechanisms} of PF against ALD

We further verified the pharmacological effects and the prediction mechanisms of PF against ALD based on the rat model. As shown in Figure 6A, ALT and AST levels were enhanced in the ethanol-treated group in comparison with the control group, whereas that of ALP decreased significantly $(\mathrm{P}<0.05)$. Conversely, supplementation of PF could effectively reduce the activities of AST and ALT as well as increase the level of ALP $(p<0.05)$. Similarly, tiopronin reduced the content of ALT $(\mathrm{p}<0.05)$, while there was no statistical difference in the AST and ALP activities $(\mathrm{p}>0.05)$. H\&E staining results indicated that PF and tiopronin markedly alleviated ethanol-triggered microvesicular steatosis with mild swelling and nuclear deviation, red blood cells overflow as well as certain lipid vacuolation in hepatocytes (Figure $6 B)$.

A

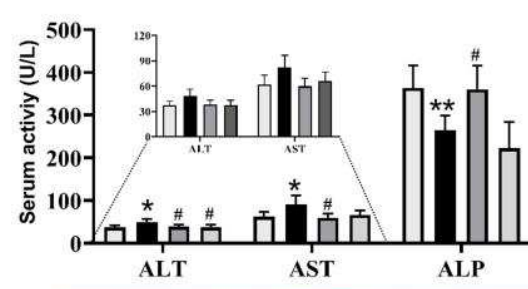

B

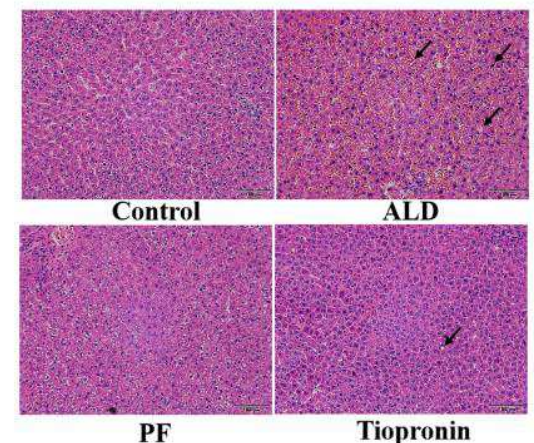

C
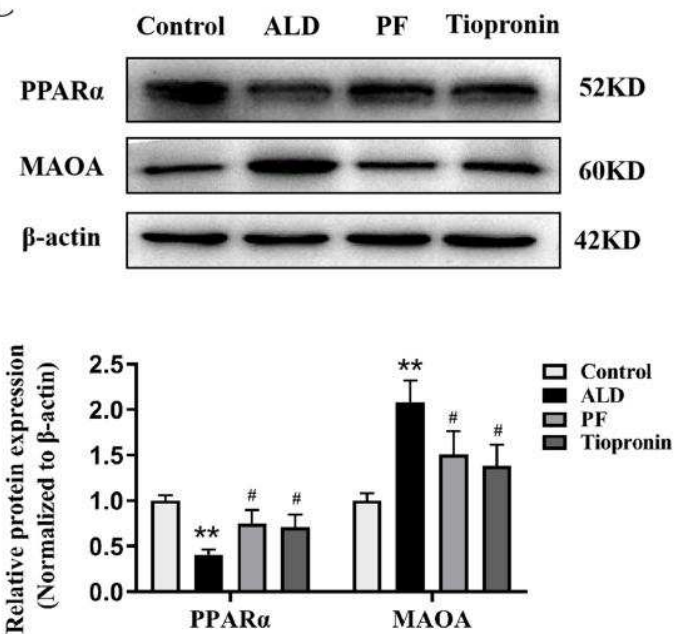

Figure 6 Protective effect of PF on ethanol induced rat liver injury

(A) Serum ALT, AST and ALP were measured using the automatic biochemistry analyzer. (B) Histological changes were observed using H\&E staining $(100 \times)$. The arrow indicates swelling 
and nuclear deviation, red blood cells overflow or lipid vacuolation. (C) Effects of PF on PPAR $\alpha$ and MAOA protein levels in liver tissue based on the western blotting assay; Data are presented as the mean $\pm \mathrm{SD}(n=3),{ }^{*} p<0.05,{ }^{*} p<0.01$ versus control group. ${ }^{*} p<0.05$ versus model group.

To validate whether the targets MAOA and PPAR $\alpha$ were involved in the protective effect of ALD by PF, their protein levels in liver tissues were examined. As shown in Figure $6 \mathrm{C}$, the expression levels of MAOA and PPAR $\alpha$ proteins were respective increased and decreased in the ALD model rats, compared to the normal control group $(\mathrm{p}<0.01)$. As hypothesized, treatment with PF significantly recovered the protein expression of MAOA and PPARacompared to the model group $(\mathrm{p}<0.05)$. Notably, the effect of PF was approximately equal to that of tiopronin. The verification results are consistent with the prediction results.

\section{Discussion}

In general, $\mathrm{PF}$ is given as tea drinks or compounded preparations with other herbmedicines by oral administration in the daily life or clinic therapy. Thus, exploring the constituents absorbed and excreted in vivo can narrow the screening scope of effective forms and build a foundation for follow-up mechanism research of PF in treating various diseases. In the present study, a global metabolic profile of PF including the identification and classification was provided by detecting the metabolites in rat plasma, urine, bile and feces after orally administration owing to high sensitivity of UPLC-QTOF-MS system. Moreover, the relative content of the prototypes and metabolites in the four biological samples were determined to evaluate the contribution of different metabolic reactions in vivo. As shown in Table 1, two categories of the parent compounds and related metabolites, including flavonoids and saponins were observed in rat biological samples. The saponins were only detected in urine and feces in the form of prototypes or metabolites that were produced from phase I metabolism, such as hydroxylation, dehydroxylation, methylation, demethylation and reduction. While the flavonoids were generally speculated to be the bioactive components, and the effective forms should be absorbed into blood with appropriate concentrations. 
If we evaluate the contribution of different metabolic reactions to the metabolite formation according to their number and relative content, we could find that sulfation and glucuronidation are both major metabolic reactions for PF flavonoids. The route of in vivo metabolism or biotransformation for flavonoids is a process to transform them into more hydrophilic metabolites, which not only enhanced their oral absorption and bioavailability, but also enhanced the excretion from the body via the bile and urine. O'Leary et al [19] demonstrated that the isoflavone conjugates formed at intestinal are easy to be transported into the hepatocytes and then excreted into the bile. Moreover, hepatic uptake and efflux transporters on the basolateral membrane participate in drug elimination, which can facilitate endogenous compounds and metabolites with poor membrane permeability to transport into hepatocytes [20]. Except for some conjugated metabolites that eluted in bile, deconjugated by intestinal microflora and excreted to feces, the others were reabsorbed in vivo, and then underwent enterohepatic circulation. Therefore, the metabolites with higher relative content in bile were also selected to follow-up mechanism analysis.

Network pharmacology combined with docking analysis revealed that Te-7XG (P6) and three metabolites genistein-7G-4'S (M11), Te-7XG-4'S (M16), tectoridin-4'S (M17) may be the effective forms in the treatment of PF on ALD. The latter three belong to conjugated metabolites. Over the past two decades, several studies showed that glucuronides, sulfates or bis-conjugates were the major existent form of flavonoids in systemic circulation and closely associated with their pharmacological actions. Our previous study found that Te-7S (M55) and Te-7G (M36) exhibited stronger inhibitory activity against aldose reductase than Te (P15) [11]. In addition, several flavonoids conjugates, such as daidzein-7-glucuronide-4'-sulfate, daidzein-4',7disulfate, apigenin-7-O-glucuronide, quercetin-3- $O$-glucuronide, quercetin-4'- $O$-glucuronide, quercetin-3'-O-sulfate and luteolin-7-O-glucuronide, have also been proved to possess some pharmacological activities, including anti-inflammatory [21], anti-oxidative [22], anti-tumor [23] and triglyceride-lowering effects [24]. Therefore, these Phase II metabolites may be responsible for the pharmacological and medicinal properties of flavonoids in vivo, and the conjugation site should be considered as their benefit for structural modification.

When compare the difference in connecting targets, Te-7XG (P6), Te-7XG-4'S (M16) and tectoridin-4'S (M17) were strongly associated with fatty acid metabolism, adipocytokine 
signaling pathway, arginine and proline metabolism, NOD-like receptor signaling pathway and glycolysis/gluconeogenesis via targeting ALDH2 and TNF. Te-7XG (P6) was also involved in NOD-like receptor signaling pathway and glycolysis/gluconeogenesis via regulating the candidate targets HSP90A1 and TPI1. Remarkably, GOT2 and CHKB, which involve in the regulation of lipid and amino acid metabolism, were only found to be related with the metabolites genistein-7G-4'S (M11), Te-7XG-4'S (M16) and tectoridin-4'S (M17). These results showed that protective role of PF depended on the interacting and synergetic of both prototypes and metabolites. The prototypes are more likely to act on glycolysis/gluconeogenesis as well as inflammation and immune regulation, while the metabolites are highly involved in regulation of lipid and amino acid metabolism.

ALD is characterized by oxidative stress, inflammation, disturbance of hepatocyte metabolism as well as bacterial translocation [25]. In the present study, KEGG enrichment analysis showed that the targets were regulated by 16 genes correlated with multiple biological process inclusive of 9 pathways, which were interacting and synergetic. Combined with docking results, the regulation of lipid and amino acid metabolism should highly involve in the protective effect of PF against ALD (Figure 7).

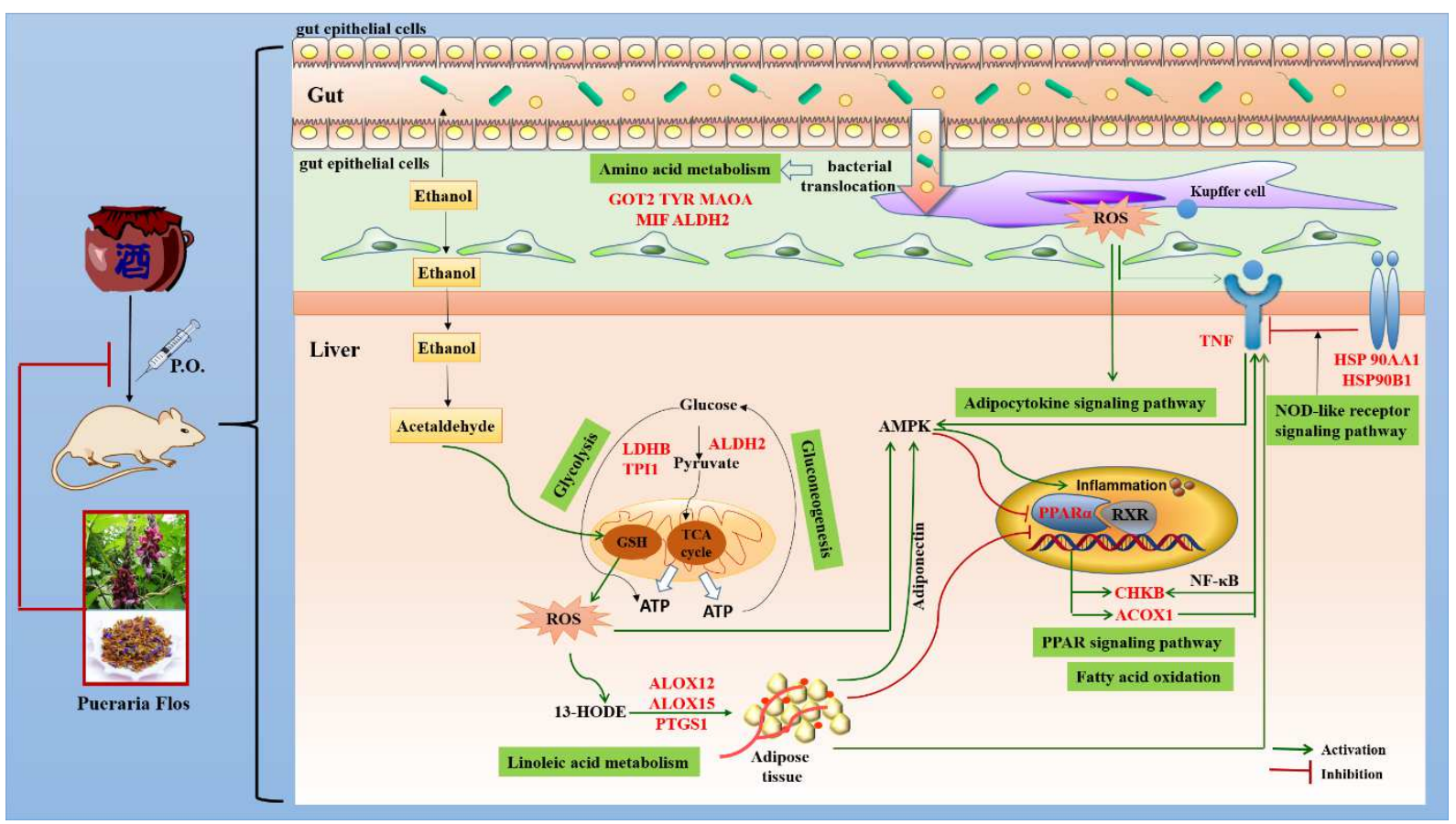

Figure 7. Potential molecular mechanism of PF acting on ALD 
Growing evidence has demonstrated that the development of ALD is associated with disturbance of lipid regulation. Fatty acid metabolism (hsa00071), adipocytokine signaling pathway (hsa04920) and PPAR signaling pathway (hsa03320), selected on the basis of the enrichment by 5 genes ACOX1, CHKB, ALDH2, PPARA and TNF, participate in the lipid regulation process of PF. PPAR $\alpha$, a key nuclear transcription factor abundantly expressed in liver, plays a major role in lipid metabolism regulation. Several studies reported that PPAR $\alpha$ agonists were expected to be a treatment for ALD by reducing oxidative stress, regulating fatty acid synthesis and oxidation, inhibiting the production and release of inflammatory and profibrogenic factors, and alleviating liver tissue damage [26]. As the target genes of PPAR $\alpha$, the expression of peroxisomal acyl-coenzyme A oxidase 1 (ACOX1) and choline/ethanolamine kinase (CHKB) through fatty acid oxidation will be reduced, the accumulation of triglyceride in liver and the content of TNF- $\alpha$ in serum was accordingly increased in the development and progression of ALD. Concordantly, increased TNF affect the expression of CHKB through the NF-kB pathway and induced inflammation, which aggravate the course of disease.

Abnormal amino acid metabolism, including tyrosine metabolism (hsa00350), phenylalanine metabolism (hsa00360) as well as arginine and proline metabolism (hsa00330), is closely related to intestinal flora metabolism, and bacterial translocation from the gut microbiota into the portal blood stream is an important driver of ALD [27]. MAOA, a mitochondrial enzyme exists mainly in the human liver, kidney, small intestine and nerve tissues, could remove the metabolites of tyrosine and phenylalanine by oxidative deamination. It had been closely associated with neurological and psychiatric disorders due to its degradation of catecholamine neurotransmitter. Recent studies provide insights into the application of MAOA as a novel predictor of clinical outcomes that MAOA expression was negatively correlated with alcohol consumption level and hepatocellular carcinoma [28]. Increasing MAOA expression or enzyme activity may be a new approach that can be used for AFL treatment. In addition, researchers founded that ALDH2, the aldehyde dehydrogenase located at mitochondrial, was the downstream gene of MAOA. A link between capacity to reduce alcohol consumption and increase liver ratio of MAO to ALDH2 has been established by animal model [28]. Other targets, such as GOT2, TYR and MIF, are also involved in the gut bacterial pathway for dopamine and 
tyrosine metabolism, which affect the intestinal permeability and bacterial translocation.

An increasing number of studies have shown that inflammation and immune regulation was also critical for the progression of ALD. Heat shock protein 90 (hsp90), an emerging therapeutic target in ALD, was a main effector in the NOD-like receptor signaling pathway (hsa04621). It is involved in initiation of the early phases of ER stress contributing to stimulation and accumulation of hepatic lipids [29]. Hsp90 inhibitors could alleviate serum ALT, endotoxin and proinflammatory cytokines such as TNF- $\alpha$ in acute and chronic alcoholic liver injury and regulating PPAR $\alpha$ to influence fatty acid oxidation and synthesis. In addition, ALOX12, ALOX15, PTGS1 that catalytic the generation of leukotrienes and prostaglandins by arachidonic acid metabolism (hsa00590), also participate in the processes of AFL through activation of inflammatory responses [30]. Their chemical inhibitors have been confirmed to significantly alleviate alcohol-induced oxidative stress, lipid accumulation and liver damage. Besides, LDHB, TPI1, ALDH2 that facts as master regulatory gene of glycolysis/gluconeogenesis (hsa00010) due to the increased cells' need for oxygen by chronic alcohol consumption, also possesses vital role in the treatment of PF in ALD.

In this study, network pharmacology and bioinformatics method were combined to elucidate the active components and mechanism of PF in the treatment of ALD. Some of the predicted results are confirmed by in vivo experiments, which preliminarily proves the scientific nature of this method. In addition, some of the compound-target-pathway interactions predicted by the network pharmacology analysis have also been confirmed in the previous studies. For example, protective effects of genistein against chronic alcohol-induced liver injury in mice was related to regulate expression of inflammatory-related factors TNF $\alpha, N F-\kappa B$ and PTGS1 [31]. Tectoridin, a characteristic isoflavone glycoside found in PF and Belamcanda chinensis (L.) DC., could protect against ethanol-induced liver steatosis mainly through modulating the disturbance of PPAR $\alpha$ pathway and ameliorating mitochondrial function [1]. Besides, the attenuate alcoholism by daidzin has been proved to be associated with liver mitochondrial MAO-ALDH2 pathway [29]. However, other constituents and targets especially conjugated metabolites predicted in the present study still need to be validated in the follow-up study. 


\section{Conclusions}

In this paper, the metabolic profile of PF in vivo was systematically described based on UPLC-QTOF-MS technology for the first time. A total of 25 prototype compounds and 82 metabolites were identified and characterized, which involved in such major metabolic pathways as glucuronidation, sulfation, methylation, hydroxylation and reduction. Furthermore, the absorbed constituent-target-pathway-disease network and docking analysis revealed that 4 metabolic components Te-7XG, genistein-7G-4'S, tectoridin-4'S and Te-7XG-4'S, 2 targets MAOA and PPARA, and 6 pathways related to lipid regulation and amino acid metabolism may play crucial roles in the PF mediated protection against ALD. Furthermore, the protective effects and predicted mechanism of PF associated with decreased and elevated expression of MAOA and PPAR $\alpha$ in ALD models were also validated. The present study not only offer novel insights into the effective form and molecular mechanism of PF against ALD, but also make beneficial exploration in the investigating the pharmacodynamic material basis of Traditional Chinese Medicine.

\section{Abbreviations}

ACOX1, Peroxisomal acyl-coenzyme A oxidase 1; ALD, alcoholic liver disease; ALDH2, Aldehyde dehydrogenase; ALOX12, Polyunsaturated fatty acid lipoxygenase ALOX12; ALOX15, Polyunsaturated fatty acid lipoxygenase ALOX15; CHKB, Choline/ethanolamine kinase; GOT2, Aspartate aminotransferase; HSP90AA1, Heat shock protein HSP 90-alpha; HSP90B1, Endoplasmin; LDHB, L-lactate dehydrogenase B chain; MAOA, Amine oxidase [flavin-containing] A; MIF, Macrophage migration inhibitory factor; PTGS1, Prostaglandin G/H synthase 1; PPARA, Peroxisome proliferator-activated receptor alpha; TNF, Tumor necrosis factor; TPI1, Triosephosphate isomerase; TYR, Tyrosinase; UPLC, ultra performance liquid chromatography.

\section{Supplementary Information}

Figure S1 Representative base peak chromatogram (BPC) of 7 mixed reference compounds (A) and PF extract (B) in the negative ion mode. See Supplemental Table 1 for the peak numbers, 
and see section 2.4 UPLC-QTOF-MS analysis for UPLC-QTOF-MS conditions.

Table S1 Compounds identified from the flower of Pueraria thomsonii Benth extract by UPLC-QTOF/MS method.

Table S2 Potential targets of 13 absorbed constitutents from Pueraria Flos.

Table S3 Therapeutic targets of alcoholic liver disease.

\section{Acknowledgement}

Not applicable

\section{Author's contribution}

Jia-lin Qu: Experimental works, Project administration, Data curation, Writing- original draft. Qiu-yue Chen: Experimental works, Data curation. Tian-fu Wei: Bioinformatics investigation of network pharmacology and molecular docking. Ning Dou: Experimental works. Dong Shang: Writing - review \& editing. Dan Yuan: Research design and supervision, Writing review \& editing.

\section{Funding}

This work was financially supported by grants from National Natural Science Foundation of China (No. 81703675), China Postdoctoral Science Foundation (2021M692398), Natural Science Foundation of Liaoning Province (No. 2019-ZD-0631), Distinguished Professor Foundation in 2011 from Liaoning Province China (No.511260) and the Leading Talent of Hundred, Thousand and Ten Thousand Project of Xingliao Gifted Person Program of Liaoning Province (No. XLYC1905013).

\section{Declarations}

\section{Ethics approval and consent to participate}

The experimental procedures were approved by the Ethics Review Committee for Animal Experimentation of Shenyang Pharmaceutical University (License number: SCXK (Liao) 20150001). 


\section{Consent for publication}

Not applicable.

\section{Competing interests}

All authors have no financial or scientific conflicts of interest with regard to the research described in this manuscript.

\section{Availability of data and materials}

Not applicable.

\section{Reference}

[1] Xiong Y, Yang YQ, Yang J, Chai HY, Li Y, Yang J, et al Tectoridin, an isoflavone glycoside from the flower of Pueraria lobata, prevents acute ethanol-induced liver steatosis in mice, Toxicology. 2010; 276(1), 64-72.

[2] Lee KT, Sohn IC, Kim DH, Choi JW, Kwon SH, Park HJ. Hypoglycemic and hypolipidemic effects of tectorigenin and kaikasaponin III in the streptozotocin-induced diabetic rat and their antioxidant activity in vitro, Arch. Pharm. Res. 2000; 23(5), 461-6.

[3] Shin JE, Bae EA, Lee YC, Ma JY, Kim DH. Estrogenic effect of main components kakkalide and tectoridin of Puerariae Flos and their metabolites. Biol. Pharm. Bull. 2006; 29(6), 1202-6.

[4] Yuan D, Xie YY, Bai X, Wu X, Yang JY, Wu CF. Inhibitory activity of isoflavones of Pueraria flowers on nitric oxide production from lipopolysaccharide-activated primary rat microglia, J. Asian Nat. Prod. Res. 2009; 11(6): 471-81.

[5] Lu J, Xie YY, Tan Y, Qu JL, Matsuda H, Yoshikawa M, et al. Simultaneous determination of isoflavones, saponins and flavones in Flos Puerariae by ultra performance liquid chromatography coupled with quadrupole time-of flight mass spectrometry. Chem. Pharm. Bull. 2013; 61(9): 941-51.

[6] Hirayama K, Matsuzuka Y, Kamiya T, Ikeguchi M, Takagaki K, Itoh K. Metabolism of isoflavones found in the Pueraria thomsonii flower by human intestinal microbiota. Biosci. Microflora. 2011; 30(4): 135-40.

[7] Bai X, Qu JL, Liu J, Sun JH, Yuan D. Isolation and identification of urinary metabolites of 
tectoridin in rats. J. Asian Nat. Prod. Res. 2011; 13(7): 604-10.

[8] Bai X, Qu JL, Lu J, Kano Y, Yuan D. Pharmacokinetics of kakkalide and its main metabolites in rat plasma determined by HPLC-DAD and LC-MS ${ }^{\mathrm{n}}$. J. Chromatogr. B 2011; 879(5-6): 395-402.

[9] Bai X, Xie YY, Liu J, Qu JL, Kano Y, Yuan D. Isolation and identification of urinary metabolites of kakkalide in rats. Drug Metab. Dispos. 2010; 38(2): 281-6.

[10]Qu JL, Gao J, Sun, JH, Zhang L, Makino T, Yuan D. Pharmacokinetics of conjugated metabolites in rat plasma after oral administration of tectoridin, J. Chromatogr. B 2012; 902: 61-9.

[11]Qu JL, Wu ZZ, Gao J, Wen H, Wang T, Yuan D. Excretion of tectoridin metabolites in rat urine and bile orally administrated at different dosages and their inhibitory activity against aldose reductase. Fitoterapia. 2014; 99: 99-108.

[12] Wang S, Gong TX, Lu J, Kano Y, Yuan D. Simultaneous determination of tectorigenin and its metabolites in rat plasma by ultra-performance liquid chromatography/quadrupole timeof-flight mass spectrometry. J. Chromatogr. B 2013; 933: 50-58.

[13]Zhang GZ, Gong TX, Kano Y, Yuan D. Metabolic profile of irisolidone in rats obtained by ultra-high performance liquid chromatography/quadrupole time-of-flight mass spectrometry. J. Chromatogr. B 2013; 941: 1-9.

[14]Kinjo J, Aoki K, Okawa M, Shii Y, Hirakawa T, Nohara T, et al. HPLC profile analysis of hepatoprotective oleanene-glucuronides in Puerariae Flos. Chem. Pharm. Bull. 1999; 47(5): 708-10.

[15]Zhang GF, Wu X, Bai X, Kano Y, Uno T, Yuan D. Simultaneous determination of four isoflavone compounds in flowers of Pueraria lobata by HPLC. Journal of Shenyang Pharmaceutical University. 2009; 26(1): 43-7.

[16] Gao K, Yu W, Yang J, Duan XH. CYP3A1/2 and 2C9/10 in rat liver microsomes mediate

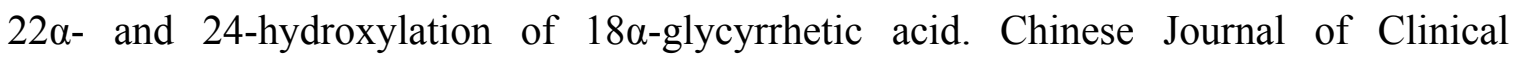
Pharmacology and Therapeutics. 2007; 12(11): 1255-60.

[17]Liang QD, He MZ, Ouyang H, Huang WP, Guo YC, Feng YL, et al. Analysis on in vivo metabolites of $\alpha$-hederin in rats by UPLC-MS/MS. Chinese Traditional and Herbal Drugs. 2014; 45(13), 1883-8. 
[18] Yu P, Qiu H, Wang M, Tian Y, Zhang Z, Song R. In vitro metabolism study of saikosaponin $\mathrm{d}$ and its derivatives in rat liver microsomes. Xenobiotica. 2016; 47(1): 1-9.

[19]O'Leary KA, Day AJ, Needs PW, Mellon FA, O'Brien NM, Williamson G. Metabolism of quercetin-7- and quercetin-3-glucuronides by an in vitro hepatic model: the role of human $\beta$-glucuronidase, sulfotransferase, catechol-O-methyltransferase and multi-resistant protein 2 (MRP2) in flavonoid metabolism. Biochem. Pharmacol. 2003; 65(3): 479-91.

[20]Funk C. The role of hepatic transporters in drug elimination. Expert Opin. Drug Metab. Toxicol. 2008; 4(4): 363-79.

[21]Min YS, Yim SH, Bai KL, Choi HJ, Jeong JH, Song HJ, et al. The inhibitory effect of quercetin-3-O- $\beta$-D-glucuronopyranoside on gastritis and reflux esophagitis in Rats. Korean J. Physiol. Pharmacol. 2009; 13(4): 295-300.

[22] Moon JH, Tsushida T, Nakahara K, Terao J. Identification of quercetin 3-O- $\beta$-Dglucuronide as an antioxidative metabolite in rat plasma after oral administration of quercetin. Free Radic. Biol. Med. 2001; 30(11): 1274-85.

[23]Chuang CH, Yeh CL, Yeh SL, Lin ES, Wang LY, Wang YH. Quercetin metabolites inhibit MMP-2 expression in A549 lung cancer cells by PPAR- $\gamma$ associated mechanisms. J. Nutri. Biochem. 2016; 33: 45-53.

[24]Eseberri I, Miranda J, Lasa A, Mosqueda-Solís A, González-Manzano S, Santos-Buelga C, et al. Effects of quercetin metabolites on triglyceride metabolism of 3T3-L1 preadipocytes and mature adipocytes. Int. J. Mol. Sci. 2019; 20(2): 264.

[25]Louvet A, Mathurin P. Alcoholic liver disease: mechanisms of injury and targeted treatment, Nat. Rev. Gastroenterol. Hepatol. 2015; 12(4): 231-42.

[26] Kersten SIntegrated physiology and systems biology of PPAR $\alpha$, Mol. Metab. 2014; 3(4): $354-71$.

[27] Vassallo G, Mirijello A, Ferrulli A, Antonelli M, Landolfi R, Gasbarrini A, et al. Review article: alcohol and gut microbiota - the possible role of gut microbiota modulation in the treatment of alcoholic liver disease. Aliment. Pharmacol. Ther. 2015; 41(10): 917-27.

[28]Cervera-Juanes R, Wilhem LJ, Park B, Lee R, Locke J, Helms C, et al. MAOA expression predicts vulnerability for alcohol use. Mol. Psychiatry. 2016; 21(4): 472-9.

[29] Ambade A, Catalano D, Lim A, Kopoyan A, Shaffer SA, Mandrekar P. Inhibition of heat 
shock protein 90 alleviates steatosis and macrophage activation in murine alcoholic liver injury. J. Hepatol. 2014; 61(4): 903-11.

[30]Zhang WL, Zhong W, Sun Q, Sun XG, Zhou ZX. Hepatic overproduction of 13-HODE due to ALOX15 upregulation contributes to alcohol-induced liver injury in mice. Sci. Rep. 2017; 7(1): 8976.

[31]Zhao L, Wang Y, Liu J, Wang K, Guo XX, Ji BP, et al. Protective effects of genistein and puerarin against chronic alcohol-induced liver injury in mice via antioxidant, antiinflammatory, and anti-apoptotic mechanisms. J. Agric. Food Chem. 2016; 64(38): 72917. 


\section{Supplementary Files}

This is a list of supplementary files associated with this preprint. Click to download.

- SupplementalFigure1.tif

- SupplementalTable1.docx

- SupplementalTable2.xlsx

- SupplementalTable3.xlsx 